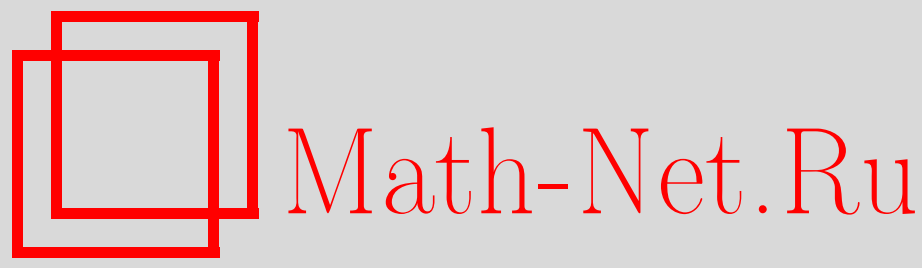

В. В. Пупышев, Приближение эффективного радиуса в задаче двумерного рассеяния центральным короткодействующим потенциалом, ТМФ, 2015, том 182, номер 2, 315-337

DOI: https://doi.org/10.4213/tmf8738

Использование Общероссийского математического портала Math-Net.Ru подразумевает, что вы прочитали и согласны с пользовательским соглашением http: //www . mathnet.ru/rus/agreement

Параметры загрузки:

IP : 35.174 .16 .151

26 апреля 2023 г., 17:11:18

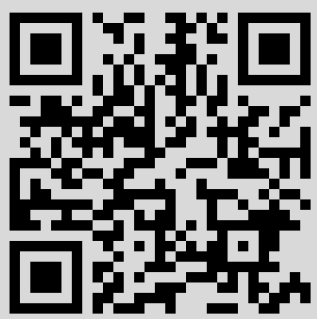




\title{
ФИЗИКА
}

Том 182, № 2

февраль, 2015

(C) 2015 г.

В. В. Пупышев*

\section{ПРИБЛИЖЕНИЕ ЭФФЕКТИВНОГО РАДИУСА В ЗАДАЧЕ ДВУМЕРНОГО РАССЕЯНИЯ ЦЕНТРАЛЬНЫМ КОРОТКОДЕЙСТВУЮЩИМ ПОТЕНЦИАЛОМ}

\begin{abstract}
Исследуется роль слабосвязанных и околопороговых резонансных состояний квантовой частицы в ее двумерном упругом низкоэнергетическом рассеянии. Энергии таких состояний определяются в приближении эффективного радиуса через корни трансцендентных уравнений. Это же приближение используется для анализа рассеяния. Получены и исследованы явные низкоэнергетические асимптотики всех парциальных фаз и сечений. Эти асимптотики содержат энергии слабосвязанных или околопороговых резонансных состояний.
\end{abstract}

Ключевые слова: двумерное рассеяние, короткодействующий потенциал, слабосвязанные и околопороговые резонансные состояния.

DOI: $10.4213 / \operatorname{tmf} 8738$

\section{1. ВВЕДЕНИЕ}

Предположим, что квантовая частица p $_{1}$ движется в трехмерном координатном пространстве $\mathcal{R}^{3}$ в поле неподвижного силового центра $O$, который воздействует на нее посредством центрального короткодействующего потенциала. Как известно [1]-[3], в этом случае сохраняющимися квантовыми числами являются волновое число $k$ и угловой момент $\ell=0,1, \ldots$ частицы $\mathrm{p}_{1}$. Функция эффективного радиуса [1], [3] для состояния рассеяния $|k, \ell\rangle$ этой же частицы в трехмерном координатном пространстве $\mathcal{R}^{3}$ определяется через парциальную фазу $\delta_{\ell}(k)$ рассеяния формулой

$$
K^{\mathrm{s}}(k) \equiv k^{2 \ell+1} \operatorname{ctg} \delta_{\ell}(k)=-\frac{1}{a^{\mathrm{s}}}+\frac{k^{2}}{2} r_{\mathrm{eff}}^{\mathrm{s}}+\cdots .
$$

Приближением эффективного радиуса [1], [3] принято называть аппроксимацию функции $K^{\mathrm{s}}(k)$ суммой двух первых слагаемых ее разложения в бесконечный ряд по четным степеням аргумента $k$. Такая сумма содержит две константы: длину рассеяния $a^{\mathrm{s}}$ и эффективный радиус $r_{\mathrm{eff}}^{\mathrm{s}}$.

*Объединенный институт ядерных исследований, Дубна, Московская обл., Россия. E-mail: pupyshev@theor.jinr.ru 
В ядерную физику низких энергий приближение эффективного радиуса для случая $S$-волнового $(\ell=0)$ рассеяния короткодействующим потенциалом ввел Бете [4]. Изящное обобщение этого приближения для произвольного значения углового момента $\ell$ предложено Друкаревым [5]. Надежный способ, предназначенный для вычисления констант $a^{\mathrm{s}}$ и $r_{\mathrm{eff}}^{\mathrm{s}}$ при любом значении $\ell$ и основанный на нелинейной версии метода фазовых функций, подробно изложен Бабиковым в его монографии [3]. Как показано в недавних работах [6]-[8], для расчета этих констант удобнее использовать рекуррентную цепочку не зависящих от энергии линейных дифференциальных уравнений первого порядка, которую нетрудно вывести, стартуя с линейной версии метода фазовых функций [3].

Обобщение приближения эффективного радиуса на случай суперпозиции кулоновского и короткодействующего потенциалов начато работой [9] Ландау и Смородинского. Обширный список исследований, инициированных этой работой и посвященных способам вычислений кулон-ядерных длины рассеяния и эффективного радиуса, дан в обзорах низкоэнергетических приближений в ядерной физике [10], [11].

Одним из традиционных приложений приближения эффективного радиуса в теоретической и экспериментальной ядерной физике низких энергий является решение задачи экстраполяции парциальных фаз $\delta_{\ell}(k)$ и соответствующих сечений в область сверхнизких энергий [10], [11], недоступных для экспериментального исследования. Другая не менее важная прикладная задача ядерной физики низких энергий и ядерной астрофизики заключается в определении вершинных констант [12], [13] процессов развала легкого ядра $A$ на два фрагмента $B$ и $C$. В работах [14]-[17] для решения этой задачи успешно использовался метод, основанный на аналитическом продолжении одноканальной функции эффективного радиуса $K^{\mathrm{s}}(k)$ в точку полюса парциальной амплитуды рассеяния двух ядер-фрагментов $B$ и $C$, отвечающую их связанному состоянию $A$. В недавних работах [18]-[20] предложено обобщение этого метода на случай двух каналов и на случай суперпозиции кулоновского и короткодействующего (ядерного) взаимодействий. Использование такого обобщения представляется перспективным подходом к исследованию ядерных реакций, которые протекают при столкновении протонов, каналируемых в кристаллической среде, с легкими ядрами, имплантированными в нее. Ожидаемое увеличение скорости протекания таких реакций основано на давно известном и экспериментально подтвержденном эффекте каналирования заряженных частиц в кристалле [21], [22], физически прозрачных качественных соображениях [23] и на двух теоретически предсказанных эффектах: эффекте сверхфокусировки [24] и эффекте резонансного каналирования пучка заряженных частиц в кристалле [25].

В работе [23] показано, что волновая функция столкновения ядра, движущегося вдоль кристаллографической оси, с ядром, имплантированным в кристалл, подчиняется двумерному уравнению Шредингера. В системе центра масс сталкивающихся ядер такую волновую функцию можно интерпретировать как волновую функцию квантовой частицы $\mathrm{p}_{1}$ с массой $m_{1}$, равной приведенной массе этих ядер, которая движется как вдоль кристаллографической оси, так и в перпендикулярной этой оси двумерной плоскости $\mathcal{P}$ трехмерного координатного пространства $\mathcal{R}^{3}$ этой же частицы.

Как известно [1], [26]-[36], движение медленной квантовой частицы в двумерной плоскости $\mathcal{P}$ имеет две особенности по сравнению с ее движением в трехмерном 
пространстве. Во-первых, в поле любого сколь угодно слабого, но притягивающего потенциала, убывающего быстрее кулоновского, квантовая частица имеет по крайней мере одно слабосвязанное состояние [1], [26], [36]. Во-вторых, сечение рассеяния квантовой частицы любым короткодействующим, в том числе и финитным, потенциалом неограниченно возрастает в пределе ее нулевой полной энергии [1], [27], [28], [34], причем старшее слагаемое асимптотики сечения в этом пределе никак не зависит от потенциала и описывается неаналитической функцией энергии, равной ее логарифму. Эту же логарифмическую функцию энергии содержат и все известные к настоящему времени определения [29]-[35] функции эффективного радиуса и длины двумерного рассеяния квантовой частицы центральным короткодействующим потенциалом.

Как отмечалось в работах [37]-[39], наличие слабосвязанного состояния квантовой частицы, движущейся в двумерной плоскости, существенно меняет сечение ее низкоэнергетического упругого рассеяния.

Анализ столкновения двух легких ядер в плоскости, перпендикулярной каналируемому пучку, является нашей конечной целью и представляется особо интересным из-за перечисленных выше существенных отличий двумерного рассеяния по сравнению с трехмерным. Достижение этой цели невозможно без последовательного построения теории двумерного низкоэнергетического рассеяния, которое логично начать с детального решения наиболее простой задачи о движении медленной квантовой частицы в поле центрального короткодействующего потенциала. Теоретическое исследование такого движения удобно разбить на три этапа.

Первый этап заключается в создании асимптотического в пределе низких энергий метода, предназначенного для вычисления всех парциальных фаз, сечений и радиальных волновых функций упругого рассеяния квантовой частицы. Этот этап преодолен в работе [34]. В ней использовалось приближение эффективного радиуса, содержащее два параметра: длину и эффективный радиус двумерного рассеяния квантовой частицы центральным короткодействующим потенциалом. Наиболее экономичные способы вычисления этих параметров предложены в работе [35].

Второй этап является более сложным и состоит в анализе условий, достаточных для существования слабосвязанных и околопороговых резонансных состояний квантовой частицы. Такой анализ дан в работе [36].

Третий этап представляется особенно интересным для практических приложений и заключается в исследовании особенностей низкоэнергетического поведения всех парциальных фаз и сечений двумерного упругого рассеяния квантовой частицы, которые порождаются ее слабосвязанными и околопороговыми резонансными состояниями. Решению этой задачи в рамках двумерного аналога [34]-[36] приближения эффективного радиуса посвящена настоящая работа.

Все используемые в работе особо значимые определения и известные соотношения собраны в разделе 2. В разделе 3 дан анализ околопороговых особенностей парциальных фаз и сечений упругого рассеяния, порожденных присутствием особых состояний рассеяния, слабосвязанных и околопороговых резонансных состояний. В заключении суммируются основные результаты выполненного анализа и обсуждаются возможные способы их обобщения и применения. 


\section{2. ОПРЕДЕЛЕНИЯ И КЛЮЧЕВЫЕ СООТНОШЕНИЯ}

Поясним обозначения и определения, принятые в предыдущих работах [34]-[36], а затем перечислим доказанные в этих же работах утверждения и соотношения.

Начнем с физических предположений. Полагаем, что квантовая частица $\mathrm{p}_{1}$ движется лишь в двумерной плоскости $\mathcal{P}$ ее координатного пространства $\mathcal{R}^{3}$. Считаем, что некоторая неподвижная точка $O$ этой плоскости является силовым центром, воздействующим на частицу $\mathrm{p}_{1}$ посредством потенциала $V$, который зависит только от обезразмеренного расстояния $x$ между точкой $O$ и этой частицей и подчинен следующим условиям:

$$
\lim _{x \rightarrow 0} x^{2}|V(x)|=0, \quad V(x) \in C^{0}(0, \infty), \quad \lim _{x \rightarrow \infty} x^{n}|V(x)|=0, \quad n=0,1,2, \ldots .
$$

Такой потенциал принято называть центральным, слабосингулярным в нуле, непрерывным на всей полуоси $x>0$ и короткодействующим. Вследствие перечисленных выше свойств потенциала $V(x)$ полный набор квантовых чисел частицы $\mathrm{p}_{1}$ состоит из ее обезразмеренного волнового числа $q$ и полуцелого числа $\lambda=-1 / 2,1 / 2, \ldots$, а радиальная волновая функция $u_{\lambda}(x ; q)=\langle x \mid q, \lambda\rangle$ физического состояния $|q, \lambda\rangle$ этой частицы определяется как регулярное (ограниченное на всей полуоси $x>0$ ) решение одномерного уравнения Шредингера

$$
\left[\partial_{x}^{2}+q^{2}-\lambda(\lambda+1) x^{-2}-V(x)\right] u_{\lambda}(x ; q)=0, \quad x>0,
$$

с граничным условием $u_{\lambda}(x ; q) \sim(q x)^{\lambda+1}$ при $q x \rightarrow 0$ и вполне определенной асимптотикой в области $q x /|\lambda| \gg 1$. Все регулярные на полуоси $x>0$ решения уравнения (2) с таким начальным условием и комплексным параметром $q$ имеют асимптотику

$$
u_{\lambda}(x ; q) \rightarrow N(q)\left[A^{+}(q) e^{i q x}-A^{-} e^{-i q x}\right], \quad \frac{|q| x}{|\lambda|} \rightarrow \infty,
$$

где

$$
A^{ \pm}(q) \equiv \frac{1}{2 i} e^{\mp i\left[\pi \lambda / 2-\delta_{\lambda}(q)\right]},
$$

а $N(q)$ и $\delta_{\lambda}(q)$ - некоторые комплекснозначные функции аргумента $q$. При условиях (1) такие решения существуют, если $q>0$ или $q=i p$, где $p \geqslant 0$. Это утверждение останется в силе, если для некоторых положительных чисел $q_{0}$ и $q_{1}$ положить $q=q_{1}-i q_{2}, q_{2} \in\left(0, q_{0}\right]$, и вместо последнего из условий (1) потребовать суммируемость функции $|V(x)| e^{2 q_{0} x}$ в области $x \gg 1$. Далее считается, что $q_{0} \equiv 2 e^{-\gamma}=1.122918 \ldots$, где $\gamma-$ константа Эйлера.

Из множества всех регулярных решений с асимптотиками (3) радиальная волновая функция $u_{\lambda}$ состояния упругого рассеяния $|q, \lambda\rangle$ выделяется условиями $q>0$ и $N(q)=1$. Эта функция имеет асимптотику

$$
u_{\lambda}(x ; q) \rightarrow \sin \left(q x-\frac{\pi \lambda}{2}+\delta_{\lambda}(q)\right), \quad \frac{|q| x}{|\lambda|} \rightarrow \infty .
$$

Величины $\delta_{\lambda}(q)$ и $\sigma_{\lambda}(q)$ называются парциальными фазой и сечением упругого рассеяния в состоянии $|q, \lambda\rangle$. Сечение $\sigma_{\lambda}(q)$ вычисляется по формулам

$$
\sigma_{\lambda}(q) \equiv \frac{\sigma_{\lambda}^{\mathrm{u}}(q)}{\operatorname{ctg}^{2} \delta_{\lambda}(q)+1}, \quad \sigma_{\lambda}^{\mathrm{u}}(q) \equiv \frac{4}{q}\left(2-\delta_{2 \lambda,-1}\right) .
$$


Здесь и далее $\delta_{i, j}$ - символ Кронекера. Сечение $\sigma_{\lambda}(q)$ достигает своего унитарного предела $\sigma_{\lambda}^{\mathrm{u}}(q)$, если волновое число $q$ равно положительному корню $p$ уравнения $\operatorname{ctg} \delta_{\lambda}(p)=0$, а фаза $\delta_{\lambda}(p)$ кратна числу $\pi / 2$. Состояние рассеяния $|p, \lambda\rangle$ с таким волновым числом считаем особым. Предел $q \rightarrow+0$ назовем пределом низких энергий. Нулевое значение безразмерной энергии рассеяния $E=q^{2}$ называем пороговым, потому что при переходе через него в область $E<0$ состояния рассеяния исчезают.

Связанные состояния выделяются из всех остальных регулярных решений совокупностью условий $q=i p, p \geqslant 0$,

$$
u_{\lambda}(x ; i p) \sim e^{-p x} \quad \text { при } \quad \frac{p x}{|\lambda|} \rightarrow \infty, \quad \int_{0}^{\infty}\left|u_{\lambda}(x)\right|^{2} d x=1,
$$

и поэтому существуют тогда и только тогда, когда волновое число $q$ равно корню $i p, p>0$, уравнения $\operatorname{ctg} \delta_{\lambda}(i p)=i$. В связанном состоянии $|i p, \lambda\rangle$ частица $\mathrm{p}_{1}$ имеет отрицательную или нулевую полную энергию $E=-p^{2}$, которой соответствует неотрицательная энергия связи $B \equiv-E=p^{2}$.

Резонансное состояние частицы $\mathrm{p}_{1}$ определяется как состояние $|q, \lambda\rangle$ с комплексным волновым числом $q=q_{1}-i q_{2}$, где $q_{1}>0, q_{2} \in\left(0, q_{0}\right)$, которому отвечает регулярное решение $u_{\lambda}$, обладающее асимптотикой в виде расходящейся круговой волны:

$$
\langle x \mid q, \lambda\rangle=u_{\lambda}\left(x ; q_{1}-i q_{2}\right) \rightarrow e^{q_{2} x+i\left[q_{1} x-\pi \lambda / 2+\delta_{\lambda}(q)\right]}, \quad \frac{|q| x}{|\lambda|} \rightarrow \infty .
$$

Асимптотика (3) общего регулярного решения совпадает с такой асимптотикой тогда и только тогда, когда $N(q)=1$, а волновое число $q$ равно корню $q_{1}-i q_{2}$ уравнения $\operatorname{ctg} \delta_{\lambda}\left(q_{1}-i q_{2}\right)=i$, где $q_{1}>0, q_{2} \in\left(0, q_{0}\right)$. Это уравнение определяет весь спектр резонансных состояний квантовой частицы $\mathrm{p}_{1}$. По определению $q=q_{1}-i q_{2}$ принадлежит четвертому квадранту комплексной плоскости волновых чисел. В этом квадранте удобно использовать специальные полярные координаты $(p, \omega)$ - модуль $p=|q|$ комплексного волнового числа $q$ и угол $\omega$, отсчитывающийся от полуоси положительных вещественных значений по часовой стрелке и определенный следующими формулами:

$$
q=p e^{i(2 \pi-\omega)}=p e^{-i \omega}, \quad \omega \equiv \operatorname{arctg} \frac{q_{2}}{q_{1}} \in(0, \pi / 2) .
$$

В координатах $(p, \omega)$ полная безразмерная энергия $E$ резонансного состояния $|q, \lambda\rangle=$ $|(p, \omega), \lambda\rangle$ представляется через энергию резонанса $E_{\mathrm{r}}$ и его ширину $\Gamma$ формулами

$$
E \equiv q^{2}=E_{\mathrm{r}}-i \frac{\Gamma}{2}, \quad E_{\mathrm{r}} \equiv q_{1}^{2}-q_{2}^{2}=p^{2} \cos 2 \omega, \quad \Gamma \equiv 4 q_{1} q_{2}=2 p^{2} \sin 2 \omega .
$$

Слабосвязанными мы считаем связанные состояния $|i p, \lambda\rangle$ с $0<p \ll q_{0}$, а состояния $|q, \lambda\rangle$ с $|q| \ll q_{0}$ называем околопороговыми состояниями упругого $(q>0)$ или резонансного $\left(q=q_{1}-i q_{2}\right)$ рассеяния.

При условиях (1) для каждого состояния рассеяния $|\lambda, q\rangle, q>0$, с выбранным значением $\lambda$ функция эффективного радиуса $K(q)$ является рядом по четным степеням волнового числа $q$ и вводится соотношениями

$$
K(q) \equiv q^{2 \lambda+1}\left[\operatorname{ctg} \delta_{\lambda}(q)-h(q)\right]=-\frac{1}{a}+\frac{q^{2}}{2} r_{\mathrm{eff}}+O\left(\frac{|q|}{q_{0}}\right)^{4} .
$$


Здесь и всюду далее

$$
h(q) \equiv \frac{2}{\pi}\left(\ln \frac{q}{2}+\gamma\right)=\frac{2}{\pi} \ln \frac{q}{q_{0}},
$$

а коэффициенты $a$ и $r_{\text {eff }}$ называются длиной рассеяния и эффективным радиусом.

Особые случаи $a=0$ и $a= \pm \infty$ в настоящей работе не рассматриваются: всюду далее считается, что $0<|a|<\infty$. В заключении поясняется, как обобщить все полученные в разделе 3 приближения парциальных фаз и сечений на два особых случая $a=0$ и $|a|=\infty$, детально исследованных в работе [35].

Как показано в работе [36], если в дополнение к условиям (1) потребовать суммируемость функции $|V(x)| e^{2 q_{0} x}$ при $x \gg 1$, то представления (7) можно использовать как аналитическое продолжение функции эффективного радиуса в область $\mathcal{A} \equiv\left\{q:|\arg | \leqslant \pi / 2, \operatorname{Im} q \geqslant-q_{0}\right\}$ комплексной плоскости волнового числа $q$. Такая суммируемость подразумевается в п. 3.3 , посвященном резонансному рассеянию.

При любых допустимых значениях числа $\lambda$, длины рассеяния $a \neq 0, \pm \infty$ и эффективного радиуса $r_{\text {eff }} \neq \pm \infty$ под приближением эффективного радиуса в области $\mathcal{A}$ мы подразумеваем замену функции $K(q)$ суммой $-1 / a+\left(q^{2} / 2\right) r_{\text {eff. }}$ В этом приближении функция $\operatorname{ctg} \delta_{\lambda}(q)$ определяется формулой

$$
\operatorname{ctg} \delta_{\lambda}(q) \approx h(q)+q^{-2 \lambda-1}\left(-\frac{1}{a}+\frac{q^{2}}{2} r_{\text {eff }}\right), \quad 0<|q| \ll q_{0},
$$

а сечение упругого рассеяния (7) - формулой

$$
\sigma_{\lambda}(q) \approx \frac{4\left(2-\delta_{2 \lambda,-1}\right) a^{2} q^{4 \lambda+1}}{\left[1-\left(q^{2} / 2\right) a r_{\mathrm{eff}}-a q^{2 \lambda+1} h(q)\right]^{2}+a^{2} q^{4 \lambda+2}}, \quad 0<q \ll q_{0} .
$$

В случае $2 \lambda=-1$ и $q / q_{0} \rightarrow 0$ приближения (9) и (10) фазы и сечения рассеяния воспроизводит их давно известные асимптотики [1]

$$
\delta_{\lambda}(q) \sim \frac{\pi / 2}{\ln \left(q / q_{0}\right)}
$$

и

$$
\sigma_{\lambda}(q) \sim \frac{4 \pi^{2}}{q} \frac{1}{\left[2 \ln \left(q / q_{0}\right)\right]^{2}+\pi^{2}} .
$$

Старшее слагаемое $(\pi / 2) / \ln q$ асимптотики фазы $\delta_{\lambda}(q)$ при $2 \lambda=-1$ и $q / q_{0} \rightarrow 0$ не зависит ни от знака, ни от формы потенциала $V(x)$. Поэтому для определения значения этой фазы в точке $q=0$ требуются дополнительные ограничения. В качестве таковых по аналогии с трехмерным рассеянием [3] будем считать, что фаза $\delta_{\lambda}(q)$ должна быть положительной или отрицательной, если в сколь угодно малой полуокрестности $\left(0, x_{0}\right), x_{0} \ll 1$, точки $x=0$ потенциал $V(x)$ является притягивающим $(V<0)$ или отталкивающим $(V>0)$ соответственно. При таком определении из представления (9) и известных свойств котангенса следует, что в любом из этих случаев фаза $\delta_{\lambda}(q)$ монотонно убывает с ростом ее аргумента, но в пределе $q \rightarrow+0$ эта фаза сходится слева к $\pi$ в первом случае и к нулю во втором.

В силу определений (5), (8) и аппроксимации (9) в приближении эффективного радиуса уравнению $\operatorname{ctg} \delta_{\lambda}(p)=0$ при $0<p \ll q_{0}$ отвечает эталонное уравнение

$$
p^{2 \lambda+1} \frac{2}{\pi} \ln \frac{p}{q_{0}}=\frac{1}{a}-\frac{1}{2} r_{\mathrm{eff}} p^{2},
$$


уравнению $\operatorname{ctg} \delta_{\lambda}(i p)=i$ при $0<p \ll q_{0}$ соответствует эталонное уравнение

$$
p^{2 \lambda+1} \frac{2}{\pi} \ln \frac{p}{q_{0}}=i^{-2 \lambda-1}\left(\frac{1}{a}+\frac{p^{2}}{2} r_{\mathrm{eff}}\right),
$$

а уравнение $\operatorname{ctg} \delta_{\lambda}\left(q_{1}-i q_{2}\right)=i$ при $|p| \ll q_{0}$ порождает систему эталонных уравнений

$$
\begin{aligned}
p^{2 \lambda+1} \frac{2}{\pi} \ln \frac{p}{q_{0}} & =\frac{1}{a} \cos [(2 \lambda+1) \omega]-\frac{p^{2}}{2} r_{\text {eff }} \cos [(2 \lambda-1) \omega], \\
p^{2 \lambda+1}\left(1+\frac{2}{\pi} \omega\right) & =-\frac{1}{a} \sin [(2 \lambda+1) \omega]+\frac{p^{2}}{2} r_{\text {eff }} \sin [(2 \lambda-1) \omega] .
\end{aligned}
$$

Завершим настоящий раздел обсуждением уравнений (12)-(15).

Заметим, что уравнения (12) и (13) имеют одинаковые левые части, а их правые части отличаются знаком первого или второго слагаемых, если число $2 \lambda+1$ не кратно или кратно четырем соответственно. Следовательно, корни $p_{1}$ и $p_{2}$ обсуждаемых уравнений (12) и (13) совпадают только при условиях $r_{\text {eff }}=0$ и $2 \lambda=-1,3,7, \ldots$. Лишь в этом случае энергия $E=p_{1}^{2}$ особого околопорогового состояния рассеяния $\left|p_{1}, \lambda\right\rangle$ равна энергии связи $B=p_{2}^{2}$ слабосвязанного состояния $\left|i p_{2}, \lambda\right\rangle$.

Выявим связь между числом корней эталонных уравнений (12) и (13).

Предположим, что значение $\lambda$ задано и уравнение (13) имеет два корня $p_{1}$ и $p_{2}$. Следовательно, существуют два слабосвязанных состояния. Положив для определенности $p_{1}<p_{2}$, найдем графическим способом число корней уравнения (12), которое по определению равно числу особых состояний рассеяния $|p, \lambda\rangle$. Пусть $2 \lambda=-1$. Уравнение (13) имеет два корня, если $a<0$ и $r_{\text {eff }}>0$. В этом случае уравнение (12) отличается от уравнения (13) лишь знаком слагаемого $p^{2} r_{\text {eff }} / 2$. Таким образом, уравнение (12) является уравнением (13), в котором $a<0$ и $r_{\text {eff }}<0$. При таких условиях это уравнение, а следовательно, и уравнение (12) имеют только один корень $p$, причем $p<p_{1}$. Теперь положим $2 \lambda=1$. Уравнение (13) имеет два корня, если $a>0$ и $r_{\text {eff }}>0$. В этом случае уравнение (12) отличается от уравнения (13) лишь знаком слагаемого $1 / a$. Поэтому уравнение (12) является уравнением (13), в котором $a<0$ и $r_{\text {eff }}>0$. При таких условиях это уравнение, а следовательно, и уравнение (12) имеют единственное решение $p$, причем $p<p_{1}$. Случай $2 \lambda \geqslant 3$ нетрудно исследовать по аналогии с выше рассмотренными случаями $2 \lambda= \pm 1$ и в итоге показать, что при $2 \lambda \geqslant 3$ уравнение (12) имеет один корень $p$, если $a>0$, и два корня $p=p_{-}$и $p=p_{+}$, если $a<0$.

Теперь предположим, что значение $\lambda$ выбрано и уравнение (12) имеет два корня $p_{1}$ и $p_{2}$. Следовательно, существуют два особых состояния рассеяния. Считая, что $p_{1}<p_{2}$, найдем графическим способом число $n$ корней уравнения (13), которое по определению равно числу слабосвязанных состояний $|i p, \lambda\rangle$. В итоге получим следующее соответствие: если $2 \lambda=-1$, то $n=1$ и $p<p_{1}$; если $2 \lambda \geqslant 1$, то $n=1$ и $p>p_{2}$ при условии $a<0$, в противном случае $n=2$.

Убедимся в том, что волновое число $p$ особого состояния рассеяния $|p, \lambda\rangle$ не может быть модулем $\left|q_{\mathrm{r}}\right|$ волнового числа околопорогового резонансного состояния $\left|q_{\mathrm{r}}, \lambda\right\rangle$. Предположим противное: пусть $p$ является решением уравнения (12) и $p=\left|q_{\mathrm{r}}\right|$. Тогда (14) сводится к уравнению, которое имеет решение только при $\omega=0$, но по определению $\omega \in(0, \pi / 2)$. В силу полученного противоречия высказанное выше утверждение верно. 


\section{3. ОКОЛОПОРОГОВЫЕ ОСОБЕННОСТИ ФАЗ И СЕЧЕНИЙ}

Первый пункт настоящего раздела является вспомогательным. В нем исследуется околопороговое поведение фазы и сечения при наличии особого состояния рассеяния. Такое исследование необходимо для того, чтобы в следующих двух пунктах выявить эффекты, порождающиеся в рассеянии только слабосвязанными или только околопороговыми резонансными состояниями.

3.1. Влияние особого состояния рассеяния. Исследуем околопороговое поведение фазы $\delta_{\lambda}(q)$ и сечения $\sigma_{\lambda}(q)$ упругого рассеяния квантовой частицы в состоянии $|q, \lambda\rangle$ при наличии ее особого состояния $|p, \lambda\rangle$, фаза которого $\delta_{\lambda}(p)$ кратна числу $\pi / 2$. Считаем, что значения $\lambda, a$ и $r_{\text {eff }}$ заданы и при этих значениях уравнение (12) имеет один корень $p$, но уравнение (13) не имеет корней на полуинтервале $(0, p+\epsilon], \epsilon / p \ll 1$, и на таком полуинтервале система уравнений (14) и (15) несовместна. Тогда имеется особое околопороговое состояние рассеяния $|p, \lambda\rangle$, но отсутствуют и слабосвязанное, и околопороговое резонансное состояния, модули волновых чисел которых меньше или равны числу $p+\epsilon$. Так как $p$ - корень уравнения (12), из приближения (9) функции $\operatorname{ctg} \delta_{\lambda}(q)$, содержащего два параметра $a$ и $r_{\text {eff }}$, можно исключить один из них. В итоге получится представление функции $\operatorname{ctg} \delta_{\lambda}(q)$ через параметры $r_{\text {eff и }} p$ :

$$
\operatorname{ctg} \delta_{\lambda}(q) \approx y_{\lambda}^{+}(q ; p)+\frac{r_{\text {eff }}}{2} \frac{q^{2}-p^{2}}{q^{2 \lambda+1}}, \quad y_{\lambda}^{+}(q ; p) \equiv h(q)-\left(\frac{p}{q}\right)^{2 \lambda+1} h(p), \quad q \ll q_{0},
$$

или представление этой же функции, но через параметры $a$ и $p$ :

$$
\operatorname{ctg} \delta_{\lambda}(q) \approx y_{\lambda}^{-}(q ; p)-\frac{1}{a p^{2}} \frac{q^{2}-p^{2}}{q^{2 \lambda+1}}, \quad y_{\lambda}^{-}(q ; p) \equiv h(q)-\left(\frac{p}{q}\right)^{2 \lambda-1} h(p), \quad q \ll q_{0} .
$$

Соответствующие таким приближениям фазу и сечение (4) обозначим символами $\delta_{\lambda}^{\mathrm{s}}(q)$ и $\sigma_{\lambda}^{\mathrm{s}}(q)$. Если особое состояние рассеяния отсутствует, сечение $\sigma_{\lambda}(q)$ определяем формулой (10).

Исследуем особый случай $2 \lambda=-1$. Согласно представлению (16) только в этом случае

$$
y_{\lambda}^{+}(q ; i p)=\frac{2}{\pi} \ln \frac{q}{p}
$$

и поэтому сечение $\sigma_{\lambda}^{\mathrm{s}}(q)$ имеет асимптотику

$$
\sigma_{\lambda}^{\mathrm{s}}(q) \sim \frac{4 \pi^{2}}{q} \frac{1}{[2 \ln (q / p)]^{2}+\pi^{2}}, \quad \frac{q}{p} \rightarrow 0 .
$$

Сравним ее с асимптотикой (11) сечения $\sigma_{\lambda}(q)$ в случае, когда особое состояние рассеяния отсутствует. Если $q<p$, то $|\ln (q / p)|<\left|\ln \left(q / q_{0}\right)\right|$, следовательно, мы имеем $\sigma_{\lambda}^{\mathrm{s}}(q)>\sigma_{\lambda}(q)$ при $q<p$. Выявим особенности поведения фазы $\delta_{\lambda}^{\mathrm{s}}(q)$ при $2 \lambda=-1$. В силу соотношения (16)

$$
\partial_{q} \delta_{\lambda}^{\mathrm{s}}(q) \approx-\left(\frac{2}{\pi q}+q r_{\mathrm{eff}}\right) \sin ^{2} \delta_{\lambda}^{\mathrm{s}}(q), \quad q \ll q_{0} .
$$


Таким образом, если $r_{\text {eff }}>0$, то производная $\partial_{q} \delta_{\lambda}^{\mathrm{s}}(q)$ отрицательна и велика по модулю, поэтому при $q / p \rightarrow 0$ фаза $\delta_{\lambda}^{\text {s }}(q)$ монотонно и быстро убывает, как $(\pi / 2) / \ln (q / p)$. При условии $r_{\text {eff }} \ll-2 / \pi q_{0}^{2}$ эта производная обращается в нуль в точке

$$
q=p_{0} \equiv \sqrt{-\frac{2}{\pi r_{\mathrm{eff}}}} \ll q_{0},
$$

поэтому в этой точке фаза $\delta_{\lambda}^{\mathrm{s}}(q)$ имеет локальный минимум.

Рассмотрим следующий особый случай $2 \lambda=1$. В силу представления (17) только в этом случае

$$
y_{\lambda}^{-}(q ; i p)=\frac{2}{\pi} \ln \frac{q}{p}
$$

и поэтому для сечения $\sigma_{\lambda}^{\mathrm{b}}(q)$ верно приближение

$$
\sigma_{\lambda}^{\mathrm{s}}(q) \approx \frac{8(\pi a)^{2} q^{3}}{\left[2 a q^{2} \ln (q / p)-\pi\left(1-q^{2} / p^{2}\right)\right]^{2}+\left(a \pi q^{2}\right)^{2}}, \quad q \ll q_{0} .
$$

Сравнив его с приближением (10) сечения $\sigma_{\lambda}(q)$, заключаем, что из неравенства $|\ln (q / p)|<\left|\ln \left(q / q_{0}\right)\right|$ вытекает, что $\sigma_{\lambda}^{\mathrm{s}}(q)>\sigma_{\lambda}(q)$ при $q<p$. Обсудим особенности поведения фазы $\delta_{\lambda}^{\mathrm{s}}(q)$ в случае $2 \lambda=1$. Вследствие соотношения (17)

$$
\partial_{q} \delta_{\lambda}^{\mathrm{s}}(q) \approx-\frac{2}{q}\left(\frac{1}{\pi}+\frac{1}{a q^{2}}\right) \sin ^{2} \delta_{\lambda}^{\mathrm{s}}(q), \quad q \ll q_{0} .
$$

Следовательно, если $a>0$, то производная $\partial_{q} \delta_{\lambda}^{\mathrm{s}}$ отрицательна и по порядку величины равна $O(-a q)$, поэтому при $q / p \rightarrow 0$ фаза $\delta_{\lambda}^{\mathrm{s}}(q)$ монотонно убывает, как $O\left(-a q^{2}\right)$. При условии $a \ll-\pi / q_{0}^{2}$ производная $\partial_{q} \delta_{\lambda}^{\text {s }}(q)$ равна нулю в точке

$$
q=p_{0} \equiv \sqrt{-\frac{\pi}{a}} \ll q_{0},
$$

поэтому в этой точке фаза $\delta_{\lambda}^{\mathrm{s}}(q)$ имеет локальный максимум.

Отметим, что в силу представления (17) фаза $\delta_{\lambda}^{\mathrm{s}}(q)$ при $2 \lambda \geqslant 3$ имеет один локальный максимум или минимум, если $a>0$ или $a<0$ соответственно.

Теперь предположим, что при данном значении $\lambda$ уравнение (12) имеет два корня $p_{1}$ и $p_{2}$, причем $p_{1}<p_{2}$. Тогда существуют два особых состояния рассеяния $\left|p_{1}, \lambda\right\rangle$ и $\left|p_{2}, \lambda\right\rangle$ с энергиями $E_{j}=p_{j}^{2}$ и фазами $\delta_{\lambda}\left(p_{j}\right), j=1,2$, кратными числу $\pi / 2$. Выведем приближенное представление функции $\operatorname{ctg} \delta_{\lambda}(q)$ для состояния рассеяния $|q, \lambda\rangle$ через его энергию $E=q^{2}$, энергии $E_{1}, E_{2}$ и константу $E_{0} \equiv q_{0}^{2}$. Для этого в уравнении (12) положим сначала $p=p_{1}$, а затем $p=p_{2}$. Два выведенных таким способом соотношения считаем системой уравнений относительно неизвестных $a$ и $r_{\text {eff. }}$ Решив эту систему, получим представления коэффициентов $a$ и $r_{\text {eff }}$ в виде функций аргументов $p_{1}$ и $p_{2}$ :

$$
\begin{aligned}
a & =\frac{p_{2}^{2}-p_{1}^{2}}{p_{1}^{2 \lambda+1} p_{2}^{2} h\left(p_{1}\right)-p_{2}^{2 \lambda+1} p_{1}^{2} h\left(p_{2}\right)}, \\
r_{\mathrm{eff}} & =2 \frac{p_{1}^{2 \lambda+1} h\left(p_{1}\right)-p_{2}^{2 \lambda+1} h\left(p_{2}\right)}{p_{2}^{2}-p_{1}^{2}} .
\end{aligned}
$$


Затем в приближении (9) заменим длину рассеяния $a$ и эффективный радиус $r_{\mathrm{eff}}$ такими функциями. В итоге получим искомое представление

$$
\operatorname{ctg} \delta_{\lambda}^{\mathrm{s}}(q) \approx \frac{1}{\pi} \ln \frac{E}{E_{0}}+\frac{1}{\pi} \sum_{j=1,2}(-1)^{j}\left(\frac{E_{j}}{E}\right)^{\lambda+1 / 2} \frac{E+E_{j}}{E_{1}-E_{2}} \ln \frac{E_{j}}{E_{0}} .
$$

Перечислим физически интересные следствия приближений (16), (17) и (21), которые порождаются наличием двух особых состояний рассеяния.

В силу этих приближений функция $\operatorname{ctg} \delta_{\lambda}^{\mathrm{s}}(q)$ ограничена, если $0<q \ll q_{0}$. Поэтому при том же условии фаза $\delta_{\lambda}^{\mathrm{s}}(q)$ не может быть нулем или числом, кратным $\pi$. Следовательно, в точках $q=p_{1}$ и $q=p_{2}$ эта фаза принимает одинаковые значения, кратные числу $\pi / 2$, а на интервале $\left(p_{1}, p_{2}\right)$ имеет по крайней мере один локальный экстремум. Если $2 \lambda=-1, a<0$ и $r_{\text {eff }}<0$, то фаза $\delta_{\lambda}^{\mathrm{s}}(q)=\pi / 2$ при $q=p_{1}, p_{2}$ и имеет локальный минимум в точке $q=p_{0} \equiv \sqrt{-2 /\left(\pi r_{\text {eff }}\right)} \in\left(p_{1}, p_{2}\right)$. В случае $2 \lambda=1, a<0$ фаза $\delta_{\lambda}^{\mathrm{s}}(q)=-\pi / 2$ при $q=p_{1}, p_{2}$ и имеет локальный максимум в точке $q=p_{0}=\sqrt{-\pi / a} \in\left(p_{1}, p_{2}\right)$. В оставшемся случае $2 \lambda \geqslant 3$ при $a<0$ фаза $\delta_{\lambda}^{\mathrm{s}}(q)=-\pi / 2$ в точках $q=p_{1}, p_{2}$ и имеет локальный максимум; если же $a>0$, то фаза $\delta_{\lambda}^{\mathrm{s}}(q)=\pi / 2$ в точках $q=p_{1}, p_{2}$ и имеет локальный минимум.

Так как в точках $q=p_{1}$ и $q=p_{2}$ фаза $\operatorname{ctg} \delta_{\lambda}^{\mathrm{s}}(q)$ одинакова и кратна числу $\pi / 2$, а в некоторой точке $p_{0} \in\left(p_{1}, p_{2}\right)$ достигает своего локального экстремума, отвечающее ей сечение $\sigma_{\lambda}^{\mathrm{s}}(q)$ равно унитарному пределу $\sigma_{\lambda}^{\mathrm{u}}(q)$ на концах отрезка $\left[p_{1}, p_{2}\right]$, а в точке $p_{0}$ имеет локальный минимум. При увеличении $\lambda$ глубина этого минимума увеличивается, потому что в этом случае второе слагаемое в представлении (16) функции $\operatorname{ctg} \delta_{\lambda}(q)$ быстро возрастает в точке $q=p_{0}$.

Стоит пояснить и проиллюстрировать практические приложения полученных выше представлений (20) и (21). Предположим, что при данном квантовом числе $\lambda$ значения волновых чисел $p_{1}, p_{2}$ или энергий $E_{1}, E_{2}$ двух особых состояний рассеяния известны. Тогда, используя представление (21), можно экстраполировать фазу и сечение рассеяния в состоянии $|q, \lambda\rangle$ в область энергий $E \ll E_{0}=q_{0}^{2}$. При том же предположении нетрудно сначала вычислить длину рассеяния и эффективный радиус по формулам (20), а затем численно исследовать уравнение (12) на наличие третьего корня $p_{3}$ и найти модуль $p_{\mathrm{b}}$ волнового числа слабосвязанного состояния $\left|i p_{\mathrm{b}}, \lambda\right\rangle$ как корень $p_{\mathrm{b}}=p$ уравнения (13).

Приведем вычисленные описанным способом значения корней $p_{3}$ и $p_{\mathrm{b}}$ в случаях $p_{1}=0.1$ и $p_{2}=0.2$. Корень $p_{3}$ существует при $2 \lambda=3$ или $2 \lambda=5$ : если $2 \lambda=3$, то $p_{3}=1.039506 \ldots$, если $2 \lambda=5$, то $p_{3}=1.120375 \ldots$. При условии $2 \lambda \leqslant 3$ имеется один корень:

$$
p_{\mathrm{b}}=\left\{\begin{array}{lll}
0.070716 \ldots, & \text { если } & 2 \lambda=-1, \\
0.282842 \ldots, & \text { если } & 2 \lambda=1, \\
1.191663 \ldots, & \text { если } & 2 \lambda=3 .
\end{array}\right.
$$

При $2 \lambda=5$ существуют два корня: $p_{\mathrm{b}}=0.227009 \ldots$ и $p_{\mathrm{b}}=1.120336 \ldots$ Для значений $p_{1}=0.1, p_{2}=0.2$ зависимость сечения $\sigma_{\lambda}^{\mathrm{s}}(q)$, вычисленного в приближении $(21)$, от квантовых чисел $q$ и $\lambda$ поясняет рис. 1 . Как видно, сечение $\sigma_{\lambda}^{\mathrm{s}}(q)$ близко к своему унитарному пределу $\sigma_{\lambda}^{\mathrm{u}}(q)$ на всем интервале $\left(p_{1}, p_{2}\right)$, если $2 \lambda=-1$, а с ростом $\lambda$ минимальное значение сечения $\sigma_{\lambda}^{\mathrm{s}}(q)$ на этом интервале быстро уменьшается. В области $q>p_{2}$ сечения $\sigma_{\lambda}^{\mathrm{s}}(q)$ при $2 \lambda=3,5$ близки к унитарному пределу, потому что 

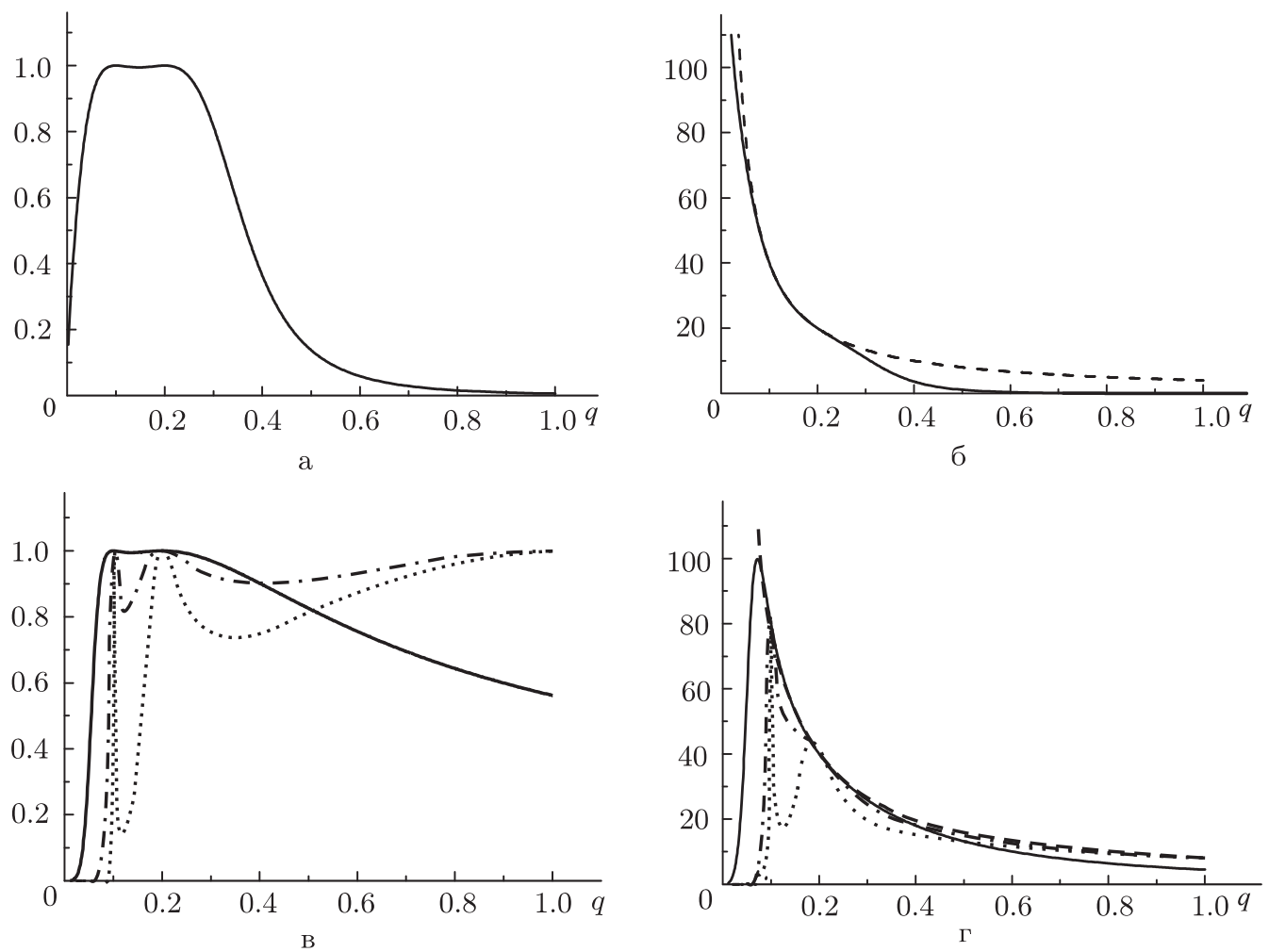

Рис. 1. Зависимость сечения $\sigma_{\lambda}^{\mathrm{s}}(q)$ от квантовых чисел $q$ и $\lambda$ при наличии особых состояний рассеяния $\left|p_{1}, \lambda\right\rangle, p_{1}=0.1$, и $\left|p_{2}, \lambda\right\rangle, p_{2}=0.2$ : отношение $\sigma_{\lambda}^{\mathrm{s}}(q) / \sigma_{\lambda}^{\mathrm{u}}(q)$ при $2 \lambda=-1(\mathrm{a})$; сечение $\sigma_{\lambda}^{\mathrm{s}}(q)$ при $2 \lambda=-1$ (б); отношения $\sigma_{\lambda}^{\mathrm{s}}(q) / \sigma_{\lambda}^{\mathrm{u}}(q)$ при $2 \lambda=1,3,5$ - сплошная, штрихпунктирная и пунктирная кривые соответственно (в); сечения $\sigma_{\lambda}^{\mathrm{s}}(q)$ при $2 \lambda=1,3,5$ - сплошная, штрихпунктирная и пунктирная кривые соответственно (г). На рис. "б” и "г" штриховая линия - унитарный предел $\sigma_{\lambda}^{\mathrm{u}}(q)$.

при выбранных значениях $p_{1}=0.1, p_{2}=0.2$ корней уравнения (12) это уравнение имеет еще один корень $p_{3}$, близкий к единице.

3.2. Рассеяние при наличии слабосвязанного состояния. Выявим особенности фаз $\delta_{\lambda}(q)$ и сечений $\sigma_{\lambda}(q)$ упругого рассеяния квантовой частицы, отличные от указанных выше, но порождающиеся ее слабосвязанными состояниями. Предположим, что значения $\lambda, a$ и $r_{\text {eff }}$ заданы и при таких значениях уравнение (13) имеет один корень $p$. Предположим также, что на полуинтервале $(0, p+\epsilon], \epsilon / p \ll 1$, уравнение (12) не имеет решений, а система уравнений (14) и (15) несовместна. Тогда имеется одно слабосвязанное состояние $|i p, \lambda\rangle$, но отсутствуют особое состояние рассеяния и резонансное состояние, модули волновых чисел которых не превосходят число $p+\epsilon$. Поэтому при $q \in(0, p+\epsilon]$ фаза $\delta_{\lambda}(q)$ состояния рассеяния $|q, \lambda\rangle$ не кратна числу $\pi / 2$. 
Исследуем поведение этой фазы и соответствующего ей сечения. Для этого используем представление (9) функции $\operatorname{ctg} \delta_{\lambda}(q)$ через параметры $a$ и $r_{\text {eff. }}$ Так как $p$ - корень уравнения (13), из этого представления можно исключить $a$ или $r_{\text {eff }}$. В результате получится приближение функции $\operatorname{ctg} \delta_{\lambda}(q)$ через параметры $r_{\text {eff }}$ и $p$,

$$
\operatorname{ctg} \delta_{\lambda}(q) \approx y_{\lambda}^{+}(q ; i p)+\frac{r_{\mathrm{eff}}}{2} \frac{q^{2}+p^{2}}{q^{2 \lambda+1}}, \quad y_{\lambda}^{+}(q ; i p) \equiv h(q)-\left(\frac{i p}{q}\right)^{2 \lambda+1} h(p),
$$

или приближение этой же функции, но через параметры $a$ и $p$,

$$
\operatorname{ctg} \delta_{\lambda}(q) \approx y_{\lambda}^{-}(q ; i p)-\frac{1}{a p^{2}} \frac{q^{2}+p^{2}}{q^{2 \lambda+1}}, \quad y_{\lambda}^{-}(q ; i p) \equiv h(q)-\left(\frac{i p}{q}\right)^{2 \lambda-1} h(p)
$$

(в обоих случаях $q \ll q_{0}$ ). Соответствующее таким приближениям сечение (4) обозначим символом $\sigma_{\lambda}^{\mathrm{b}}(q)$. Примем обозначения $a_{\mathrm{b}} \equiv a, r_{\mathrm{b}} \equiv r_{\mathrm{eff}}$ и $\delta_{\lambda}^{\mathrm{b}}(q) \equiv \delta_{\lambda}(q)$. Если слабосвязанное состояние отсутствует, используем прежние обозначения $a, r_{\text {eff }}$ и $\delta_{\lambda}(q)$, а сечение $\sigma_{\lambda}(q)$ определим формулой (10). Заметим, что верны оба неравенства $a_{\mathrm{b}} \neq a, r_{\mathrm{b}} \neq r_{\mathrm{eff}}$ или по крайней мере одно из них.

Предположим, что число $2 \lambda+1$ кратно четырем. Используем представления (22).

Случай $2 \lambda=-1$ является исключительным: только в этом случае

$$
y_{\lambda}^{+}(q ; i p)=\frac{2}{\pi} \ln \frac{q}{p}
$$

и поэтому сечение $\sigma_{\lambda}^{\mathrm{b}}(q)$ имеет асимптотику

$$
\sigma_{\lambda}^{\mathrm{b}}(q) \sim \frac{4 \pi^{2}}{q} \frac{1}{[2 \ln (q / p)]^{2}+\pi^{2}}=\frac{1}{\sqrt{E}} \frac{4 \pi^{2}}{\ln ^{2}(E / B)+\pi^{2}}, \quad \frac{q}{p} \rightarrow 0 .
$$

Сравним ее с асимптотикой (11) сечения $\sigma_{\lambda}(q)$ в случае, когда слабосвязанное состояние отсутствует. Если $q<p$, то $|\ln (q / p)|<\left|\ln \left(q / q_{0}\right)\right|$, и поэтому $\sigma_{\lambda}^{\mathrm{b}}(q)>\sigma_{\lambda}(q)$ при $q<p$.

Перечислим особенности поведения фазы $\delta_{\lambda}^{\mathrm{b}}(q)$ при $2 \lambda=-1$, порожденные представлением (22). Если $r_{\mathrm{b}}>0$, то производная $\partial_{q} \delta_{\lambda}^{\mathrm{b}}(q)$ отрицательна и велика по модулю, поэтому при $q / p \rightarrow 0$ фаза $\delta_{\lambda}(q)$ монотонно и быстро убывает, как $(\pi / 2) \ln (q / p)$. При условии $r_{\mathrm{b}} \ll-2 /\left(\pi q_{0}^{2}\right)$ производная обращается в нуль в точке

$$
q=\sqrt{\frac{2}{\pi r_{\mathrm{b}}}} \ll q_{0},
$$

поэтому в той же точке фаза $\delta_{\lambda}^{\mathrm{b}}(q)$ имеет локальный минимум.

Отметим, что из приближения (22) при $2 \lambda=-1$ следует менее точное приближение

$$
\operatorname{ctg} \delta_{\lambda}^{\mathrm{b}}(q) \approx \frac{1}{\pi} \ln \frac{E}{B}, \quad \lambda=-\frac{1}{2}, \quad \frac{E}{B} \rightarrow 0,
$$

которое впервые получено в работе [37] и использовалось в недавних работах [38], [39], по-видимому, из-за того, что авторам неизвестно более хорошее приближение. 
Если $2 \lambda=3,7, \ldots$, то согласно формулам (22) в малой $\epsilon$-окрестности $(p-\epsilon, p+\epsilon)$, $0<\epsilon \ll p$, точки $p$ функция $y_{\lambda}^{+}(q ; i p)$ близка к функции $(2 / \pi) \ln (q / p)$ и равна нулю в точке $q=p$, как и в рассмотренном выше случае $2 \lambda=-1$. Поэтому в точке $q=p$

$$
\operatorname{ctg} \delta_{\lambda}^{\mathrm{b}}(q) \approx p^{1-2 \lambda} r_{\mathrm{b}}, \quad \sigma_{\lambda}^{\mathrm{b}}(q) \approx\left(2-\delta_{2 \lambda,-1}\right) \frac{4 p^{4 \lambda-3}}{p^{4 \lambda-2}+r_{\mathrm{b}}^{2}}, \quad 2 \lambda=-1,3,7, \ldots
$$

Если $\left|r_{\mathrm{b}}\right| \ll p^{2 \lambda-1}$, то в этой точке значение фазы $\delta_{\lambda}^{\mathrm{b}}(q)$ близко к числу, кратному $\pi / 2$, а сечение $\sigma_{\lambda}^{\mathrm{b}}(q)$ по порядку величины равно $O(1 / p)$ и поэтому довольно велико. Сравним такое сечение $\sigma_{\lambda}^{\mathrm{b}}(p)$ с сечением $\sigma_{\lambda}(q)$, когда $2 \lambda=3,7, \ldots$. По формуле $(10)$ находим $\sigma_{\lambda}(q) \approx 8 a^{2} p^{4 \lambda+1}$. Следовательно, отношение $\sigma_{\lambda}^{\mathrm{b}}(p) / \sigma_{\lambda}(p)$ по порядку величины равно $O\left(1 / p^{4 \lambda+2}\right)$ и поэтому велико и возрастает при увеличении числа $\lambda$.

Предположим, что число $2 \lambda+1$ не кратно четырем. Используем формулы (23).

Случай $2 \lambda=1$ является особым: только в этом случае

$$
y_{\lambda}^{-}(q ; i p)=\frac{2}{\pi} \ln \frac{q}{p},
$$

и поэтому для сечения $\sigma_{\lambda}^{\mathrm{b}}(q)$ верно приближение

$$
\sigma_{\lambda}^{\mathrm{b}}(q) \approx \frac{8\left(\pi a_{\mathrm{b}}\right)^{2} q^{3}}{\left[2 a_{\mathrm{b}} q^{2} \ln (q / p)-\pi\left(1+q^{2} / p^{2}\right)\right]^{2}+\left(a_{\mathrm{b}} \pi q^{2}\right)^{2}}, \quad q \ll q_{0} .
$$

Сравнив его с приближением (10) сечения $\sigma_{\lambda}(q)$, заключаем, что при $q<p$ из неравенства $|\ln (q / p)|<\left|\ln \left(q / q_{0}\right)\right|$ следует соотношение $\sigma_{\lambda}^{\mathrm{b}}(q)>\sigma_{\lambda}(q)$.

Обсудим особенности поведения фазы $\delta_{\lambda}^{\mathrm{b}}(q)$ в случае $2 \lambda=1$, обусловленные приближением (23). Если $a_{\mathrm{b}}>0$, то производная $\partial_{q} \delta_{\lambda}^{\mathrm{b}}$ отрицательна и по порядку величины равна $O\left(-a_{\mathrm{b}} q\right)$, поэтому фаза $\delta_{\lambda}^{\mathrm{b}}(q)$ монотонно убывает, как $O\left(-a_{\mathrm{b}} q^{2}\right)$. При условии $a_{\mathrm{b}} \ll-\pi / q_{0}^{2}$ производная $\partial_{q} \delta_{\lambda}^{\mathrm{b}}(q)$ равна нулю в точке

$$
q=\sqrt{-\frac{\pi}{a_{\mathrm{b}}}} \ll q_{0}
$$

поэтому в этой точке фаза $\delta_{\lambda}^{\mathrm{b}}(q)$ имеет локальный максимум.

Пусть $2 \lambda=5,9, \ldots$. Тогда согласно $(22)$ в малой $\epsilon$-окрестности $(p-\epsilon, p+\epsilon)$, $\epsilon \ll p$, точки $p$ функция $y_{\lambda}^{+}(q ; i p)$ близка к функции $(2 / \pi) \ln (q / p)$ и равна нулю в точке $q=p$, как и в исследованном выше случае $2 \lambda=1$. Поэтому в точке $q=p$ верны следующие аппроксимации:

$$
\operatorname{ctg} \delta_{\lambda}^{\mathrm{b}}(q) \approx-\frac{2}{a_{\mathrm{b}}} p^{-2 \lambda-1}, \quad \sigma_{\lambda}^{\mathrm{b}}(q) \approx 8 \frac{a_{\mathrm{b}}^{2} p^{4 \lambda+1}}{a_{\mathrm{b}}^{2} p^{4 \lambda+2}+4}, \quad 2 \lambda=1,5,9, \ldots
$$

Если $\left|a_{\mathrm{b}}\right| \gg 2 / p^{2 \lambda+1}$, то в этой точке значение фазы $\delta_{\lambda}^{\mathrm{b}}(q)$ близко к числу, кратному $\pi / 2$, а сечение $\sigma_{\lambda}^{\mathrm{b}}(q)$ по порядку величины равно $O(1 / p)$ и поэтому довольно велико. Сравним такое сечение $\sigma_{\lambda}^{\mathrm{b}}(p)$ с сечением $\sigma_{\lambda}(q)$ при $2 \lambda=5,9, \ldots$. По формуле $(10)$ находим $\sigma_{\lambda}(q) \approx 8 a^{2} p^{4 \lambda+1}$. Следовательно, отношение $\sigma_{\lambda}^{\mathrm{b}}(p) / \sigma_{\lambda}(p)$ по порядку величины равно $O\left(1 / p^{4 \lambda+2}\right)$ и поэтому велико и возрастает при увеличении числа $\lambda$. 
Теперь предположим, что при данном значении $\lambda$ уравнение (13) имеет два корня $p_{1}$ и $p_{2}$, отличные от корней уравнения (12). Тогда существуют два слабосвязанных состояния $\left|i p_{1}, \lambda\right\rangle$ и $\left|i p_{2}, \lambda\right\rangle$ с энергиями связи $B_{j}=p_{j}^{2}, j=1,2$. Выведем приближенное представление функции $\operatorname{ctg} \delta_{\lambda}(q)$ для состояния рассеяния $|q, \lambda\rangle$ через его энергию $E=q^{2}$, энергии связи $B_{1}, B_{2}$ и константу $E_{0} \equiv q_{0}^{2}$. Для этого в уравнении (13) положим сначала $p=p_{1}$, а затем $p=p_{2}$. Два полученных таким образом соотношения считаем системой уравнений относительно неизвестных коэффициен-

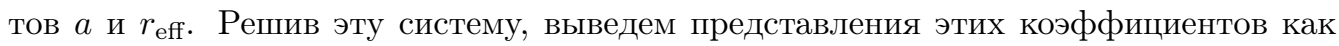
функций аргументов $p_{1}$ и $p_{2}$ :

$$
\begin{aligned}
& a_{\mathrm{b}}=\frac{p_{2}^{2}-p_{1}^{2}}{\left(i p_{1}\right)^{2 \lambda+1} p_{2}^{2} h\left(p_{1}\right)-\left(i p_{2}\right)^{2 \lambda+1} p_{1}^{2} h\left(p_{2}\right)}, \\
& r_{\mathrm{b}}=2 \frac{\left(i p_{1}\right)^{2 \lambda+1} h\left(p_{1}\right)-\left(i p_{2}\right)^{2 \lambda+1} h\left(p_{2}\right)}{p_{2}^{2}-p_{1}^{2}} .
\end{aligned}
$$

Затем в приближении (9) заменим длину рассеяния $a$ и эффективный радиус $r_{\text {eff }}$ такими функциями. В итоге получим искомое представление

$$
\operatorname{ctg} \delta_{\lambda}^{\mathrm{b}}(q) \approx \frac{1}{\pi} \ln \frac{E}{E_{0}}+\frac{1}{\pi} \sum_{j=1,2} i^{2(\lambda+j)+1}\left(\frac{B_{j}}{E}\right)^{\lambda+1 / 2} \frac{E+B_{j}}{B_{2}-B_{1}} \ln \frac{B_{j}}{E_{0}} .
$$

Сформулируем основные результаты выполненного выше анализа фаз и сечений. При любых значениях длин рассеяния $a_{\mathrm{b}}, a$ и эффективных радиусов $r_{\mathrm{b}}, r_{\mathrm{eff}}$ в области $q<p$ в случае $2 \lambda= \pm 1$ сечение $\sigma_{\lambda}^{\mathrm{b}}(q)$ околопорогового рассеяния при наличии слабосвязанного состояния $|i p, \lambda\rangle$ заметно превышает сечение рассеяния $\sigma_{\lambda}(q)$ при отсутствии такого состояния. Этот же вывод остается справедливым в случае $2 \lambda=3,7, \ldots$ или в случае $2 \lambda=5,9, \ldots$ при соответствующем дополнительном условии $\left|r_{\mathrm{b}}\right| \ll p^{2 \lambda-1}$ или $\left|a_{\mathrm{b}}\right| \gg 2 / p^{2 \lambda+1}$. Согласно работе [36] такие условия являются достаточными для существования слабосвязанного состояния, но, как показано выше, из всех таких состояний эти условия выделяют состояния, при наличии которых сечение $\sigma_{\lambda}^{\mathrm{b}}(q)$ обладает особым свойством: $\sigma_{\lambda}^{\mathrm{b}}(q)=O(1 / p)$, если $q=p$.

Поясним прикладное значение формул (25)-(29).

Используя формулы (25)-(27), можно решить две задачи: найти значения длины рассеяния $a_{\mathrm{b}}$ и эффективного радиуса $r_{\mathrm{b}}$ по измеренным значениям энергии связи $B=p^{2}$ слабосвязанного состояния $|i p, \lambda\rangle$ и сечения рассеяния $\sigma_{\lambda}^{\mathrm{b}}(p)$ при энергии $E=B$ либо предсказать оценки величин $B$ и $\sigma_{\lambda}^{\mathrm{b}}(p)$, используя предварительно вычисленные значения $a_{\mathrm{b}}$ и $r_{\mathrm{b}}$.

Предположим, что имеются два слабосвязанных состояния и известны модули $p_{1}, p_{2}$ их волновых чисел или же энергии связи $B_{1}, B_{2}$. Тогда, используя представление $(29)$, можно экстраполировать фазу $\delta_{\lambda}^{\mathrm{b}}(p)$ и сечение $\sigma_{\lambda}^{\mathrm{b}}(p)$ рассеяния в состоянии $|q, \lambda\rangle$ в область околопороговых значений энергий $E \ll E_{0}=q_{0}^{2}$. При том же предположении несложно сначала вычислить длину рассеяния и эффективный радиус по формулам (28), а затем исследовать уравнение (13) на наличие третьего корня $p_{3}$ и найти волновое число $p_{\mathrm{s}}$ особого состояния рассеяния $\left|p_{\mathrm{s}}, \lambda\right\rangle$ как корень $p_{\mathrm{s}}=p$ уравнения (12). 


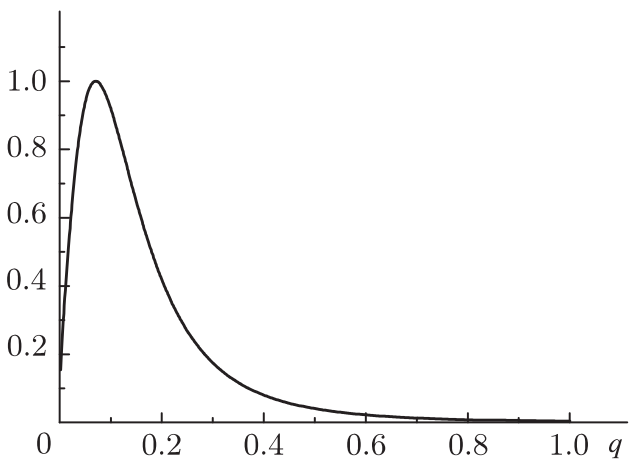

a

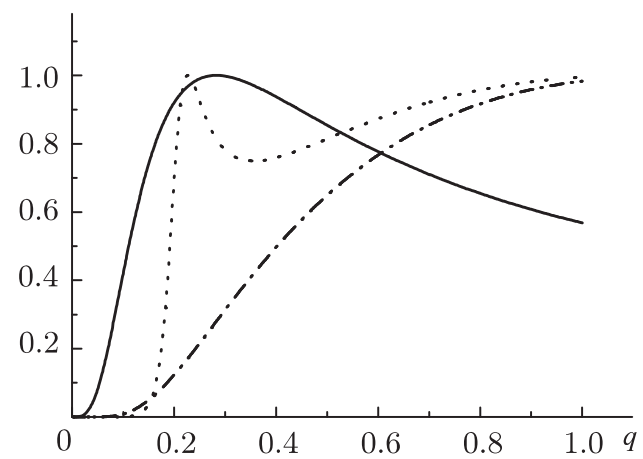

B

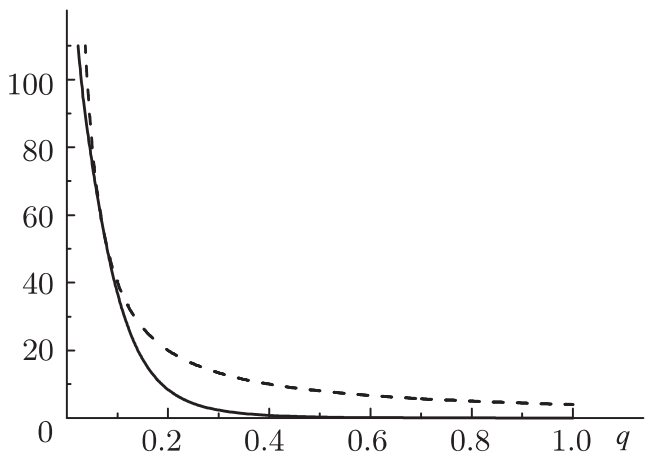

6

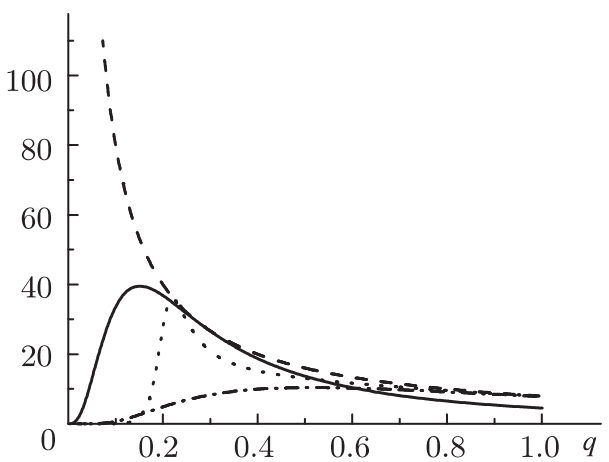

$\Gamma$

Рис. 2. Зависимость сечения $\sigma_{\lambda}^{\mathrm{b}}(q)$ от квантовых чисел $q$ и $\lambda$ при наличии слабосвязанных состояний $\left|i p_{1}, \lambda\right\rangle, p_{1}=0.1$, и $\left|i p_{2}, \lambda\right\rangle, p_{2}=0.2$ : отношение $\sigma_{\lambda}^{\mathrm{b}}(q) / \sigma_{\lambda}^{\mathrm{u}}(q)$ при $2 \lambda=-1(\mathrm{a})$; сечение $\sigma_{\lambda}^{\mathrm{b}}$ при $2 \lambda=-1$ (б); отношения $\sigma_{\lambda}^{\mathrm{b}}(q) / \sigma_{\lambda}^{\mathrm{u}}(q)$ при $2 \lambda=1,3,5$ - сплошная, штрихпунктирная и пунктирная кривые соответственно (в); сечения $\sigma_{\lambda}^{\mathrm{b}}(q)$ при $2 \lambda=1,3,5$ - сплошная, штрихпунктирная и пунктирная кривые соответственно (г). На рис. "б” и "г" штриховая линия - унитарный предел $\sigma_{\lambda}^{\mathrm{u}}(q)$.

Приведем вычисленные таким способом значения корней $p_{3}$ и $p_{\mathrm{s}}$ в случае $p_{1}=0.1$, $p_{2}=0.2$. Корень $p_{3}$ существует при условии $2 \lambda \geqslant 3$ и близок к единице: $p_{3}=$ $1.039506 \ldots$, если $2 \lambda=3$, и $p_{3}=1.120375 \ldots$, если $2 \lambda=5$. При условии $2 \lambda \leqslant 3$ имеется один корень:

$$
p_{\mathrm{s}}=\left\{\begin{array}{lll}
0.070716 \ldots, & \text { если } & 2 \lambda=-1, \\
0.282842 \ldots, & \text { если } & 2 \lambda=1, \\
1.191663 \ldots, & \text { если } & 2 \lambda=3 .
\end{array}\right.
$$

В случае $2 \lambda=5$ число корней равно двум, а их значения таковы: $p_{\mathrm{s}}=0.227100 \ldots$ и $p_{\mathrm{s}}=1.120336 \ldots$. При тех же значениях $p_{1}=0.1, p_{2}=0.2$ зависимость сечения $\sigma_{\lambda}^{\mathrm{b}}(q)$, вычисленного в приближении $(29)$, от квантовых чисел $q$ и $\lambda$ показана на 
рис. 2. Видно, что сечение $\sigma_{\lambda}^{\mathrm{b}}(q)$ не имеет особенностей при волновом числе $q$, равном модулю $p_{1}$ или $p_{2}$ первого или второго слабосвязанных состояний, и близко к своему унитарному пределу $\sigma_{\lambda}^{\mathrm{u}}(q)$ только в малой окрестности вполне определенного волнового числа $q$, совпадающего с волновым числом $p_{\mathrm{s}}$ соответствующего особого состояния рассеяния.

3.3. Резонансное рассеяние. Приступим к анализу фазы $\delta_{\lambda}(q)$ и сечения $\sigma_{\lambda}(q)$ околопорогового упругого рассеяния в состоянии $|q, \lambda\rangle$ при наличии околопорогового резонансного состояния $\left|q_{\mathrm{r}}, \lambda\right\rangle$.

Предположим, что значения $\lambda, a$ и $r_{\text {eff }}$ заданы, и при таких значениях система уравнений (14) и (15) имеет единственное решение $(p, \omega)$, но уравнения (13) и (12) не имеют корней, по крайней мере на полуинтервале $(0, p+\epsilon], \epsilon / p \ll 1$. При таких предположениях квантовая частица имеет одно околопороговое резонансное состояние $|(p, \omega), \lambda\rangle$, но модули волновых чисел ее особых состояний рассеяния и слабосвязанных состояний превышают число $p+\epsilon$.

Заметим, что из системы уравнений (14) и (15) следуют равенства

$$
\begin{aligned}
a & =-p^{-2 \lambda-1} \frac{\sin 2 \omega}{[1+(2 / \pi) \omega] \cos (2 \lambda-1) \omega+h(p) \sin (2 \lambda-1) \omega}, \\
r_{\text {eff }} & =-2 p^{2 \lambda-1} \operatorname{cosec}(2 \omega)\left\{\left(1+\frac{2}{\pi} \omega\right) \cos (2 \lambda+1) \omega+h(p) \sin (2 \lambda+1) \omega\right\} .
\end{aligned}
$$

Поэтому приближение (9) функции $\operatorname{ctg} \delta_{\lambda}(q)$ можно представить через координаты $p$ и $\omega$ волнового числа $q_{\mathrm{r}}$ резонансного состояния $\left|q_{\mathrm{r}}, \lambda\right\rangle$. Используя функцию

$$
\eta(\omega) \equiv \frac{\pi+2 \omega}{\sin 2 \omega}
$$

запишем это представление в виде трех соотношений

$$
\begin{gathered}
\operatorname{ctg} \delta_{\lambda}(q ; p, \omega) \approx h(q)-h(p) z_{\lambda}^{+}(q)-z_{\lambda}^{-}(q), \\
z_{\lambda}^{+}(q) \equiv\left(\frac{p}{q}\right)^{2 \lambda+1} \operatorname{cosec}(2 \omega)\left\{\left[\left(\frac{q}{p}\right)^{2}-1\right] \sin (2 \lambda+1) \omega+2 \sin \omega \cos 2 \lambda \omega\right\}, \\
z_{\lambda}^{-}(q) \equiv\left(\frac{p}{q}\right)^{2 \lambda+1} \eta(\omega)\left\{\left[\left(\frac{q}{p}\right)^{2}-1\right] \cos (2 \lambda+1) \omega-2 \sin \omega \sin 2 \lambda \omega\right\} .
\end{gathered}
$$

Эти соотношения позволяют определить, имеет ли фаза рассеяния $\delta_{\lambda}(q ; p, \omega)$ значения, кратные числу $\pi / 2$, или локальные экстремумы, порожденные наличием резонансного состояния. При любом заданном значении $\lambda$ решение такой задачи сводится к численному анализу простых и кратных корней уравнения $\operatorname{ctg} \delta_{\lambda}(q ; p, \omega)=0$ как функций полярных координат $p$ и $\omega$ резонансного состояния.

Для примера найдем кратный корень такого уравнения в случае $2 \lambda= \pm 1$. Заметим, что только в этом случае функция $z_{\lambda}^{+}(q)$ тождественно равна единице. Поэтому первая из формул (31) существенно упрощается и принимает вид

$$
\operatorname{ctg} \delta_{\lambda}(q ; p, \omega) \approx \frac{1}{\pi}\left\{2 \ln \frac{q}{p}+i^{2 \lambda+1} \eta(\omega)\left[\cos 2 \omega-\left(\frac{p}{q}\right)^{4 \lambda}\right]\right\} .
$$


Следовательно, сечение (4) аппроксимируется формулой

$$
\sigma_{\lambda}(q ; p, \omega) \approx \frac{1}{q} \frac{4 \pi^{2}\left(2-\delta_{2 \lambda,-1}\right)}{\left\{2 \ln (q / p)+i^{2 \lambda+1} \eta(\omega)\left[\cos 2 \omega-(p / q)^{4 \lambda}\right]\right\}^{2}+\pi^{2}},
$$

а для уравнения $\operatorname{ctg} \delta_{\lambda}(q ; p, \omega)=0$ эталонным является довольно простое уравнение

$$
2 \ln \frac{q}{p}=i^{2 \lambda+1} \eta(\omega)\left[\left(\frac{p}{q}\right)^{4 \lambda}-\cos 2 \omega\right] .
$$

Это уравнение имеет кратный корень $q=q_{\lambda} \equiv p[2 \eta(\omega)]^{\lambda}$ тогда и только тогда, когда угол $\omega$ равен корню $\omega_{\mathrm{t}}$ уравнения

$$
\ln \eta(\omega)=\eta(\omega) \cos 2 \omega-1 .
$$

Существует лишь один такой корень: $\omega=\omega_{\mathrm{t}}=0.480189 \ldots$. Следовательно, все резонансные состояния $|(p, \omega), \pm 1 / 2\rangle$ с любым значением координаты $p \ll q_{0}$, но с углом $\omega=\omega_{\mathrm{t}}$ являются особыми в следующем смысле: при наличии любого такого состояния фаза рассеяния $\delta_{\lambda}\left(q ; p, \omega_{\mathrm{t}}\right)$ в состояния рассеяния $|q, \pm 1 / 2\rangle$ имеет локальный экстремум в точке $q=q_{\lambda}=p\left[\eta\left(\omega_{\mathrm{t}}\right)\right]^{\lambda}$ - минимум или максимум, если $2 \lambda=-1$ или $2 \lambda=1$. Так как $\eta\left(\omega_{\mathrm{t}}\right)=2.140908 \ldots>1$, то $q_{\lambda}<p$, если $2 \lambda=-1$, и $q_{\lambda}>p$, если $2 \lambda=1$. В точке $q=q_{\lambda}$ равны нулю и функция $\operatorname{ctg} \delta_{\lambda}\left(q ; p, \omega_{\mathrm{t}}\right)$, и ее первая производная, а вторая производная принимает значение $2\left(2-\delta_{2 \lambda,-1}\right) / \pi q_{\lambda}^{2}$. Поэтому в малой $\epsilon$-окрестности $\left(q_{\lambda}-\epsilon, q_{\lambda}+\epsilon\right), 0<\epsilon \ll q_{\lambda}$, точки $q_{\lambda}$ верно приближение функции $\operatorname{ctg} \delta_{\lambda}\left(q ; p, \omega_{\mathrm{t}}\right), 2 \lambda= \pm 1$, частичной суммой ее ряда Тейлора с двумя нулевыми слагаемыми и третьим слагаемым, отличным от нуля:

$$
\left.\operatorname{ctg} \delta_{\lambda}\left(q ; p, \omega_{\mathrm{t}}\right) \approx \partial_{q}^{2} \operatorname{ctg} \delta_{\lambda}\left(q ; p, \omega_{\mathrm{t}}\right)\right|_{q=q_{\lambda}} \frac{\left(q-q_{\lambda}\right)^{2}}{2}=\left(2-\delta_{2 \lambda,-1}\right) \frac{\left(q / q_{\lambda}-1\right)^{2}}{\pi} .
$$

Соответствующее сечение (4) в случае $2 \lambda= \pm 1$ в обозначениях $E=q^{2}$ и $E_{\lambda} \equiv q_{\lambda}^{2}$ имеет вид

$$
\sigma_{\lambda}\left(q ; p, \omega_{\mathrm{t}}\right) \approx \sigma_{\lambda}^{t}\left(q ; q_{\lambda}\right), \quad \sigma_{\lambda}^{\mathrm{t}}\left(q ; q_{\lambda}\right) \equiv \frac{1}{\sqrt{E}} \frac{4 \pi^{2}\left(2-\delta_{2 \lambda,-1}\right)}{2^{2 \lambda+1}\left(\sqrt{E / E_{\lambda}}-1\right)^{4}+\pi^{2}} .
$$

Это сечение обладает интересной с экспериментальной точки зрения особенностью: оно близко к своему унитарному пределу в любой точке $q$ области $\left(q_{\lambda}-\epsilon, q_{\lambda}+\epsilon\right)$, $0<\epsilon \ll q_{\lambda}$, с точностью порядка $O\left(\left(q / q_{0}\right)^{2}+\left(\epsilon / q_{\lambda}\right)^{3}\right)$.

Итак, случай $2 \lambda= \pm 1$ исследован: показано, что в этом случае аппроксимации (32) и (33) справедливы при любых допустимых значениях координат $(p, \omega)$ волнового числа $q_{\mathrm{r}}$ резонансного состояния $\left|q_{\mathrm{r}}, \lambda\right\rangle$, а приближение (34) верно при любом значении координаты $p$, но при $\omega=\omega_{\mathrm{t}}$.

При $2 \lambda>1$ первая из формул (31) содержит довольно сложные функции $z_{\lambda}^{ \pm}(q)$. Поэтому простые приближения для парциальных фаз и сечений можно получить лишь в двух пределах $\omega \rightarrow+0$ и $\omega \rightarrow \pi / 2-0$. Приступим к исследованию этих пределов.

Сформулируем нашу первую задачу следующим образом: используя представление (31), найти приближение сечения (4) в малой $\epsilon$-окрестности $(p-\epsilon, p+\epsilon)$, $0<\epsilon \ll p$, точки $p$ при условии $2|\lambda| \omega \ll 1$ и любом наперед заданном значении $\lambda$. 
Прежде чем приступать к решению, стоит пояснить физический смысл условия $2|\lambda| \omega \ll 1$. Вследствие определений (5) и (6) это условие означает, что в комплексной плоскости переменной $q$ волновое число $q_{\mathrm{r}}=q_{1}-i q_{2},\left|q_{\mathrm{r}}\right|=p$, резонансного состояния $\left|q_{\mathrm{r}}, \lambda\right\rangle$ лежит вблизи положительной части вещественной полуоси $\left(q_{2} \ll q_{1}\right)$ тем ближе к оси, чем больше квантовое число $\lambda$. Поэтому ширина резонанса $\Gamma$ мала, время жизни $\hbar / \Gamma$ обсуждаемого резонансного состояния велико, а резонансная энергия $E_{\mathrm{r}}$ является положительной величиной. Такие долгоживущие резонансные состояния особенно интересны с экспериментальной точки зрения.

Перейдем к решению поставленной задачи. В формулах (31) для функций sin $2 \omega$ и $\sin 2 \lambda \omega$ мы не будем использовать аппроксимации, все остальные тригонометрические функции заменим их разложениями в ряды Тейлора с центром в точке $\omega=0$; если $2 \lambda \neq 1$, то функцию $(q / p)^{2 \lambda+1}$ аппроксимируем суммой двух первых слагаемых ее ряда Тейлора с центром в точке $q=p$. В получившемся разложении функции $\operatorname{ctg} \delta_{\lambda}(q ; p, \omega)$ оставим только те слагаемые, которые неограниченно возрастают или убывают, как $O(\epsilon / p)$ или $O(2|\lambda| \omega)$. Итоговое представление будет искомым приближением

$$
\operatorname{ctg} \delta_{\lambda}(q ; p, \omega) \approx \frac{2}{\pi} \ln \frac{q}{p}+\left[1-\left(\frac{q}{p}\right)^{2}\right] \operatorname{cosec} 2 \omega+\sin 2 \lambda \omega,
$$

которому в обозначениях (6) соответствует сечение

$$
\sigma_{\lambda}(q ; p, \omega) \approx \frac{4}{\sqrt{E}}\left(2-\delta_{2 \lambda,-1}\right) \frac{(\Gamma / 2)^{2}}{\left[E-E_{\mathrm{r}}-(\Gamma / 2) \sin 2 \lambda \omega\right]^{2}+(\Gamma / 2)^{2}} .
$$

Отметим, что полученные приближения (35) и (36) содержат функцию $\sin 2 \lambda \omega$ и верны с абсолютной точностью порядка $\left.O(2|\lambda| \omega+\epsilon / p)^{2}\right)$ для любого $\lambda$, но лишь при условиях $2|\lambda| \omega \ll 1$ и $|q-p|<\epsilon \ll p$.

Обсудим особенности, фазы и сечения, порожденные приближениями (35), (36) и функцией $\sin 2 \lambda \omega$. Значения $q_{\lambda}$ и $E_{\lambda}$ волнового числа и соответствующей энергии, при которых фаза кратна $\pi / 2$, а сечение достигает своего максимального значения, равного унитарному пределу, зависят от квантового числа $\lambda$ следующим образом:

$$
q_{\lambda} \approx p(1+\sin 2 \omega \sin 2 \lambda \omega)^{1 / 2}, \quad E_{\lambda}=q_{\lambda}^{2} \approx E_{\mathrm{r}}+\frac{\Gamma}{2} \sin 2 \lambda \omega .
$$

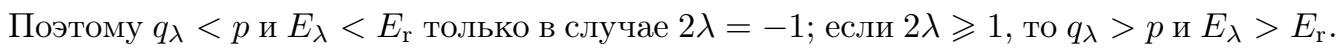
Физическая причина, порождающая указанные неравенства, заключается в том, что в исходном уравнении Шредингера (2) слагаемое $\lambda(\lambda+1) / x^{2}$ при $\lambda=-1 / 2$ является притягивающим (центростремительным) барьером, а при всех остальных значениях $\lambda$ - отталкивающим (центробежным) барьером. Неравенство $\Gamma \ll E_{\mathrm{r}}$ обуславливает пикообразное поведение сечения (36) вблизи точки $E=E_{\mathrm{r}}$ : в ее малой окрестности это сечение имеет локальный экстремум.

Наша следующая задача такова: используя формулы (31), найти приближение сечения (4) в $\epsilon$-окрестности $(p-\epsilon, p+\epsilon), 0<\epsilon \ll p$, точки $p$ при условии $|\lambda|(\pi-2 \omega) \ll 1$ и любом наперед заданном значении $\lambda$.

Выявим физические следствия ограничения $|\lambda|(\pi-2 \omega) \ll 1$ для угла $\omega$. В силу определений (5) и (6) при таком ограничении волновое число $q_{\mathrm{r}}=q_{1}-i q_{2},\left|q_{\mathrm{r}}\right|=p$, резонансного состояния $\left|q_{\mathrm{r}}, \lambda\right\rangle$ расположено в комплексной плоскости переменной $q$ вблизи отрицательной части мнимой оси $\left(q_{1} \ll q_{2}\right)$ тем ближе к оси, чем больше 
квантовое число $\lambda$. Поэтому резонансная энергия $E_{\mathrm{r}}=q_{1}^{2}-q_{2}^{2}$ является отрицательной величиной и выполняется соотношение $\Gamma \ll\left|E_{\mathrm{r}}\right|$.

Приступим к решению. В формулах (31) функцию $\sin 2 \omega$ не аппроксимируем, все остальные тригонометрические функции заменим разложениями в ряды Тейлора с центром в точке $\omega=\pi / 2$, пренебрежем слагаемыми порядка $O(|\lambda|(\pi-2 \omega)+\epsilon / p)$. В итоге получим приближение

$$
\operatorname{ctg} \delta_{\lambda}(q ; p, \omega) \approx 2 i^{2 \lambda-1}\left(\frac{p}{q}\right)^{2 \lambda+1}\left[\frac{q^{2}}{p^{2}}+1\right] \operatorname{cosec} 2 \omega,
$$

справедливое при оговоренных выше условиях $|\lambda|(\pi-2 \omega) \ll 1$ и $|q-p|<\epsilon \ll p$.

Следовательно, при тех же условиях и при любом квантовом числе $\lambda$ соответствующее сечение (4) приближается формулой

$$
\sigma_{\lambda}(q ; p, \omega) \approx \frac{4}{\sqrt{E}}\left(2-\delta_{2 \lambda,-1}\right) \frac{(\Gamma / 2)^{2}}{4\left(\left|E_{\mathrm{r}}\right| / E\right)^{2 \lambda+1}\left(E+\left|E_{\mathrm{r}}\right|\right)^{2}+(\Gamma / 2)^{2}} .
$$

Из приближения (37) следует, что вблизи точки $q=p$ фаза $\delta_{\lambda}(q ; p, \omega)$ является знакопостоянной и гладкой функцией при любом $\lambda$. Согласно формуле (38) соответствующее такой фазе сечение $\sigma_{\lambda}(q ; p, \omega)$ вблизи точки $E \approx\left|E_{\mathrm{r}}\right|$ монотонно убывает с ростом энергии $E=q^{2}$, если $2 \lambda=-1$, и монотонно возрастает, если $2 \lambda \geqslant 1$. В рассматриваемом случае резонансная энергия $E_{\mathrm{r}}$ отрицательная, поэтому и фаза $\delta_{\lambda}(q ; p, \omega)$, и сечение $\sigma_{\lambda}(q ; p, \omega)$ являются монотонными функциями и ведут себя так же, как фаза и сечение упругого рассеяния при наличии слабосвязанного состояния с энергией связи $B=\left|E_{\mathrm{r}}\right|$.

Наглядное представление о всех выявленных выше особенностях сечения околопорогового рассеяния $\sigma_{\lambda}(q ; p, \omega)$ в состояниях $|q, \pm 1 / 2\rangle$ при наличии резонансных состояний $|(p, \omega), \pm 1 / 2\rangle$ при $p=0.2$ и для разных значений угла $\omega$ дает рис. 3 . На нем сплошными кривыми изображены сечения (4), вычисленные в приближении (31).

Сплошные кривые на рис. 3а и 3в - графики сечений $\sigma_{\lambda}(q ; p, \omega)$ при небольших значениях угла $\omega=0.1, \omega=0.2$ и $\omega=0.3$ - имеют острые пики и касаются штриховых кривых, изображающих соответствующие унитарные пределы $\sigma_{\lambda}^{\mathrm{u}}(q)$ при $2 \lambda= \pm 1$. Как видно, абсцисса точки касания лежит справа или слева от точки $q=p=0.2$, если $2 \lambda=-1$ или $2 \lambda=1$ соответственно. Поведение кривых на этих рисунках полностью соответствует аппроксимации (36), полученной при условии $2|\lambda| \omega \ll 1$.

Сплошные кривые с номером 1 на рис. $3 б$ и 3 г - графики сечений $\sigma_{\lambda}(q ; p, \omega)$ в особом случае $\omega=\omega_{\mathrm{t}}$ - близки к графикам соответствующих унитарных пределов $\sigma_{\lambda}^{\mathrm{u}}(q)$ в области $0.04<q<0.14$ или $0.3<q<0.7$, если $2 \lambda=-1$ или $2 \lambda=1$ соответственно. Эта особенность подтверждает справедливость приближения (34).

Сплошные кривые с номерами 2,3 на рис. 3б и Зг изображают сечения $\sigma_{\lambda}(q ; p, \omega)$ при довольно больших значениях угла $\omega=0.8$ и $\omega=1$. Как видно, при таких углах вблизи точки $q=p=0.2$ сечение $\sigma_{\lambda}(q ; p, \omega)$ монотонно убывает в случае $2 \lambda=-1$ и монотонно возрастает в случае $2 \lambda=1$. Эти особенности сечения подтверждают приближение $(38)$, полученное при условии $|\lambda|(\pi-2 \omega) \ll 1$.

Более подробную информацию о поведении сечений $\sigma_{\lambda}(q ; p, \omega)$ при $2 \lambda= \pm 1$ в особом случае $\omega=\omega_{\mathrm{t}}$ можно извлечь из рис. 4. На нем изображены графики отношений сечений $\sigma_{\lambda}\left(q ; p, \omega_{\mathrm{t}}\right)$ и $\sigma_{\lambda}^{t}\left(q ; p, \omega_{\mathrm{t}}\right)$ к унитарному пределу $\sigma_{\lambda}^{\mathrm{u}}(q)$, вычисленные при 

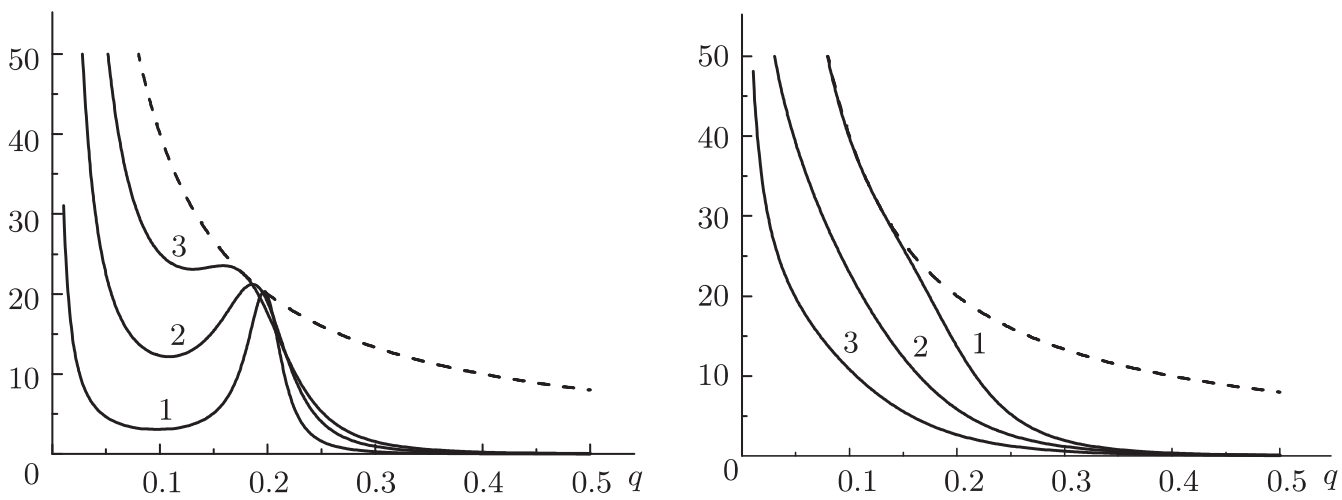

a

б
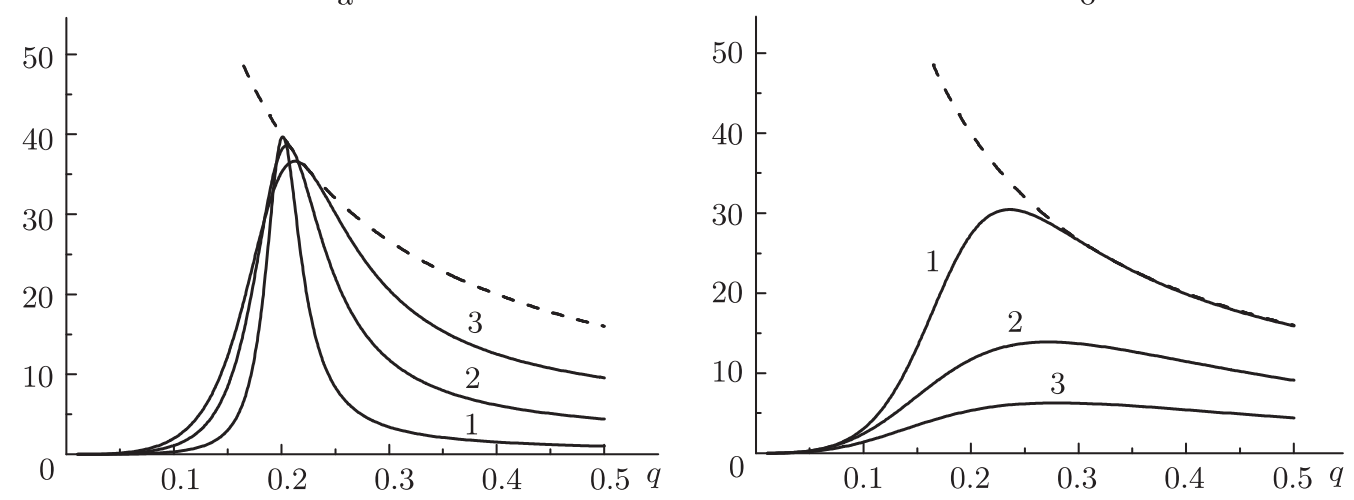

B

$\Gamma$

Рис. 3. Сечения резонансного рассеяния $\sigma_{\lambda}(q ; p, \omega), p=0.2$, в следующих случаях: при $2 \lambda=-1$ и $\omega=0.1, \omega=0.2, \omega=0.3$ - кривые $1,2,3$ (а); при $2 \lambda=-1$ и $\omega=0.480189, \omega=0.8, \omega=1-$ кривые $1,2,3$ (б); при $2 \lambda=1$ и $\omega=0.1, \omega=0.2, \omega=3$ - кривые $1,2,3$ (в); при $2 \lambda=1$ и $\omega=0.480189$, $\omega=0.8, \omega=1$ - кривые $1,2,3$ (г). Унитарные пределы $\sigma_{\lambda}^{\mathrm{u}}(q)$ при $2 \lambda= \pm 1$ на всех рисунках - штриховые кривые.

$2 \lambda= \pm 1$ и $p=0.2$ по формулам (4), (31) и $(34)$, в которых $q_{\lambda}=0.096653 \ldots<p$, если $2 \lambda=-1$, и $q_{\lambda}=0.413851 \ldots>p$, если $2 \lambda=1$. Равенство $\sigma_{\lambda}(q ; p, \omega)=\sigma_{\lambda}^{\mathrm{u}}(q)$ в случае $2 \lambda=-1$ выполняется при следующих значениях $q_{ \pm}$волнового числа $q$ : если $2 \lambda=-1$, то $q_{-} \approx 0.06$, а $q_{+} \approx 0.12$; если $2 \lambda=1$, то $q_{-} \approx 0.32$, а $q_{+} \approx 0.68$. Согласно рис. 4 приближение $\sigma_{\lambda}(q) \approx \sigma_{\lambda}^{t}(q)$ верно в области $q_{-}<q<q_{+}$, но неприменимо при малых $\left(q<q_{-}\right)$или больших $\left(q>q_{+}\right)$значениях волнового числа $q$.

\section{4. ЗАКЛЮЧЕНИЕ}

Сформулируем основные результаты настоящей работы. В рамках приближения эффективного радиуса (9), (10) впервые удалось выявить и исследовать низкоэнергетические особенности всех $(2 \lambda=-1,1,3, \ldots)$ парциальных фаз и сечений двумерного рассеяния квантовой частицы центральным короткодействующим потен- 

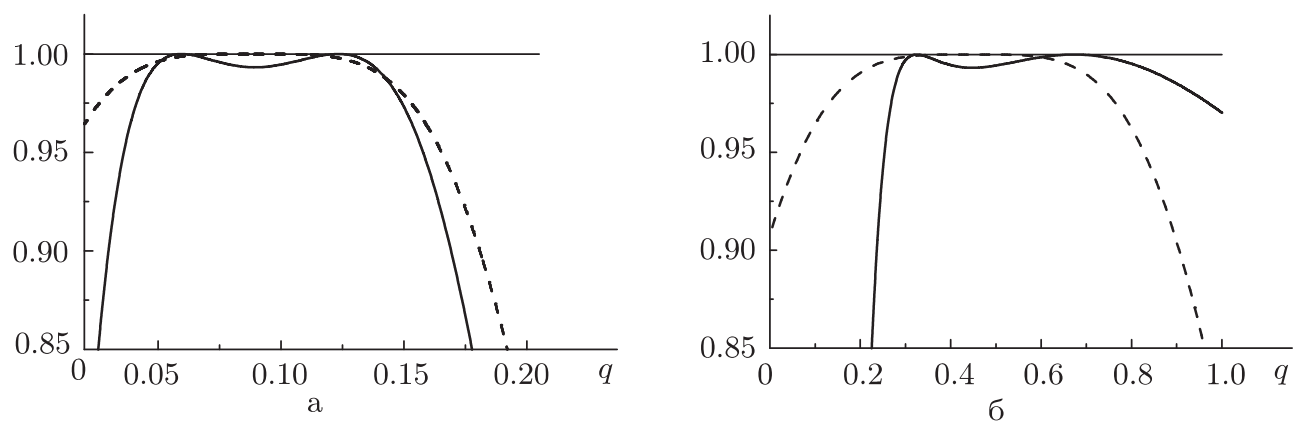

Рис. 4. Зависимость от $q$ отношений $\sigma_{\lambda}\left(q ; p, \omega_{\mathrm{t}}\right) / \sigma_{\lambda}^{\mathrm{u}}(q)$ (сплошные кривые) и $\sigma_{\lambda}^{t}\left(q ; q_{\lambda}\right) / \sigma_{\lambda}^{\mathrm{u}}(q)$ (штриховые кривые) при $p=0.2$ в случаях $2 \lambda=-1$ (а) и $2 \lambda=1$ (б).

циалом произвольной формы. В результате выполненных исследований получены удобные для практических приложений низкоэнергетические приближения парциальных фаз и сечений (16)-(38) упругого рассеяния квантовой частицы при наличии особых состояний рассеяния, слабосвязанных и околопороговых резонансных состояний. При выводе этих приближений считалось, что длина рассеяния $a$ является конечной ненулевой константой. Для полноты исследования преодолеем эти ограничения.

В случае $a=0$ асимптотическое представление (7) функции эффективного радиуса $K(q)$ теряет смысл. Как показано в работе [35], эту функцию и ее параметры $a$ и $r_{\text {eff }}$ следует переопределить и использовать функцию эффективного радиуса

$$
K^{+}(q) \equiv q^{2 \lambda+3}\left[\operatorname{ctg} \delta_{\lambda}(q)-h(q)\right]=-\frac{1}{a^{+}}+\frac{q^{2}}{2} r_{\mathrm{eff}}^{+}+\cdots
$$

с ненулевой и ограниченной по модулю длиной рассеяния $a^{+}$. Функция $K(q)$ становится функцией $K^{+}(q)$ в результате замен $\lambda \rightarrow \lambda+1, a \rightarrow a^{+}$и $r_{\text {eff }} \rightarrow r_{\text {eff }}^{+}$. Следовательно, в рассматриваемом случае $a=0$ после такой же замены в правых частях формул (16)-(38) получатся соответствующие приближения парциальных фаз и сечений упругого рассеяния квантовой частицы в состоянии $|q, \lambda\rangle$.

В случае $|a|=\infty$ согласно работе [35] вместо функции эффективного радиуса $K(q)$ следует использовать функцию эффективного радиуса

$$
K^{-}(q) \equiv q^{2 \lambda-1}\left[\operatorname{ctg} \delta_{\lambda}(q)-h(q)\right]=-\frac{1}{a^{-}}+\frac{q^{2}}{2} r_{\text {eff }}^{-}+\cdots,
$$

в которой длина рассеяния $a^{-}$удовлетворяет условию $0<\left|a^{-}\right|<\infty$. После замен $\lambda \rightarrow \lambda-1, a \rightarrow a^{-}$и $r_{\text {eff }} \rightarrow r_{\text {eff }}^{-}$функция $K(q)$ переходит в функцию $K^{-}(q)$. Поэтому в обсуждаемом случае $|a|=\infty$ нужно выполнить такую же замену в правых частях аппроксимаций (16)-(38). В итоге получатся искомые приближения парциальных фаз и сечений упругого рассеяния квантовой частицы в состоянии $|q, \lambda\rangle$.

В заключение отметим, что выполненные в настоящей работе исследования представляются актуальными в связи с бурным развитием теоретической и экспериментальной физики ультрахолодных атомарных и молекулярных газов в магнитооп- 
тических ловушках [40], [41]. Во многих из таких ловушек движение частиц газа можно с хорошей точностью считать двумерным. Для численного и качественного анализа двумерного столкновения двух частиц такого газа предлагается использовать полученные в настоящей работе низкоэнергетические аппроксимации (16)-(38).

\section{Список литературы}

[1] Л. Д. Ландау, Е. М. Лифшиц, Теоретическая физика, т. 3: Квантовая механика. Нерелятивистская теория, Наука, М., 1974.

[2] Дж. Тейлор, Теория рассеяния. Квантовая теория нерелятивистских столкновений, Мир, М., 1975.

[3] В. В. Бабиков, Метод фазовых функиий в квантовой механике, Наука, М., 1976.

[4] H. A. Bethe, Phys. Rev., 76:1 (1949), 38-50.

[5] Г. Ф. Друкарев, ЖЖЭТФ, 80:2 (1981), 537-540.

[6] S. A. Rakityansky, N. Elander, J. Phys. A: Math. Theor., 42:22 (2009), 225302, 18 pp.

[7] В. В. Пупышев, ЯФ, 76:2 (2013), 199-215.

[8] В. В. Пупышев, ЯФ, 77:5 (2014), 686-698.

[9] Л. Д. Ландау, Я. А. Смородинский, ЖЭТФ, 14 (1944), 269-275.

[10] В. В. Пупышев, О. П. Соловцова, ЭЧАЯ, 27:4 (1996), 859-929.

[11] В. В. Пупышев, ЭЧАЯ, 28:6 (1997), 1457-1528.

[12] Л. Д. Блохинцев, И. Борбей, Э.И.Долинский, ЭЧАЯ, 8:6 (1977), 1189-1245.

[13] Л. Д. Блохинцев, В. О. Еременко, ЯФ, 71:7 (2008), 1247-1253.

[14] L. D. Blokhintsev, V. I. Kukulin, A. A. Sakharuk, D. A. Savin, E. V. Kuznetsova, Phys. Rev. C, 48:5 (1993), 2390-2398.

[15] Ю. В. Орлов, Б. Ф. Иргазиев, Л. И. Никитина, ЯФ, 73:5 (2010), 787-802.

[16] J.-M. Sparenberg, P. Capel, D. Baye, Phys. Rev. C, 81:1 (2010), 011601, 4 pp.

[17] R. Yarmukhamedov, D. Baye, Phys. Rev. C, 84:2 (2011), 024603, 7 pp.

[18] Л. Д. Блохинцев, ЯФ, 74:7 (2011), 1008-1012.

[19] Л. Д. Блохинцев, Изв. РАН. Сер. физ., 76:4 (2012), 481-484.

[20] Л. Д. Блохинцев, Д. А. Савин, ЯФ, 77:3 (2014), 376-386.

[21] Й. Линдхард, УФН, 99:2 (1969), 249-296.

[22] А. М. Таратин, ЭЧАЯ, 29:5 (1998), 1063-1118.

[23] П. М. Красовицкий, Н. Ж. Такибаев, Изв. РАН. Сер. физ., 70:5 (2006), 709-712.

[24] Yu. N. Demkov, J. D. Meyer, Eur. Phys. J. B, 42:3 (2004), 361-365.

[25] O. Chuluunbaatar, A. A. Gusev, V. L. Derbov, P. M. Krassovitskiy, S. I. Vinitsky, ЯФ, 72:5 (2009), 811-821.

[26] B. Simon, Ann. Phys., 97:2 (1976), 279-288.

[27] I. R. Lapidus, Am. J. Phys., 50:1 (1982), 45-47.

[28] P. G. Averbuch, J. Phys. A: Math. Gen., 19:12 (1986), 2325-2335.

[29] B. J. Verhaar, L.P.H. de Goey, J.P.H.W. van den Eijnde, E. J. D. Vredenbregt, Phys. Rev. A, 32:3 (1985), 1424-1429.

[30] N. N. Khuri, A. Martin, J.-M. Rishard, T. T. Wu, J. Math. Phys., 50:7 (2009), 072105, 17 pp., arXiv: 0812.4054.

[31] D. Bollé, F. Gesztesy, Phys. Rev. A, 30:3 (1984), 1279-1293.

[32] S. K. Adhikari, W. G. Gibson, Phys. Rev. A, 46:7 (1992), 3967-3977.

[33] S. A. Rakityansky, N. Elander, J. Phys. A: Math. Theor., 45:13 (2012), 135209, 28 pp., arXiv: 1201.0172 . 
[34] В. В. Пупышев, ЯФ, 77:5 (2014), 699-710.

[35] В. В. Пупышев, ТМФ, 180:3 (2014), 342-367.

[36] В. В. Пупышев, ТМФ, 179:1 (2014), 102-122.

[37] M. Randeria, J.-M. Duan, L.-Y. Shieh, Phys. Rev. B, 41:1 (1990), 327-343.

[38] M. Klawunn, A. Pikovski, L. Santos, Phys. Rev. A, 82:4 (2010), 044701, 4 pp., arXiv: 1008.2444.

[39] M. Rosenkrantz, W. Bao, Phys. Rev. A., 84:5 (2011), 050701, 5 pp., arXiv: 1201.6167.

[40] P. Cladé, C. Ryu, A. Ramanathan, K. Helmerson, W. D. Phillips, Phys. Rev. Lett., 102:17 (2009), 170401, 4 pp., arXiv: 0805.3519.

[41] L. D. Caar, D. DeMille, R. V. Krems, J. Ye, New J. Phys., 11 (2009), 055049, 87 pp.

Поступила в редакцию 19.06.2014, после доработки 12.08.2014 